\title{
Radial positive definite functions and spectral theory of the Schrödinger operators with point interactions
}

\author{
N. Goloshchapova ${ }^{1, *}$, M. Malamud ${ }^{1, * *}$, and V. Zastavnyi ${ }^{2, * * *}$ \\ ${ }^{1}$ R. Luxemburg str. 74, 83114 Donetsk, Ukraine \\ ${ }^{2}$ Univesitetskaja str. 24, 83001 Donetsk, Ukraine
}

Key words Schrödinger operator, point interactions, self-adjoint extension, spectrum, positive definite function MSC (2000) 47A10, 47B25

Dedicated to the 75th anniversary of Eduard Tsekanovskii.

We complete the classical Schoenberg representation theorem for radial positive definite functions. We apply this result to study spectral properties of self-adjoint realizations of two- and three-dimensional Schrödinger operators with point interactions on a finite set. In particular, we prove that any realization has purely absolutely continuous non-negative spectrum.

Copyright line will be provided by the publisher

\section{Introduction}

An important topic in quantum mechanics is the spectral theory of Schrödinger operators on the Hilbert space $L^{2}\left(\mathbb{R}^{d}\right), d \in\{1,2,3\}$, with potentials supported on a discrete (finite or countable) set of points of $\mathbb{R}^{d}$. There is an extensive literature on such operators (see [1, 3,-5, 8, 10, 20, 22, 26, 28] and references therein). The first mathematical problem is to associate a self-adjoint operator (Hamiltonian) on $L^{2}\left(\mathbb{R}^{d}\right)$ with the differential expression

$$
\mathfrak{L}_{d}:=-\Delta+\sum_{j=1}^{m} \alpha_{j} \delta\left(\cdot-x_{j}\right), \quad \alpha_{j} \in \mathbb{R}, \quad m \in \mathbb{N} .
$$

There are at least two natural ways to associate self-adjoint operator $H_{X, \alpha}$ with the following differential expression in $L^{2}\left(\mathbb{R}^{1}\right)$

$$
\mathfrak{L}_{1}:=-\frac{\mathrm{d}^{2}}{\mathrm{dx}^{2}}+\sum_{j=1}^{m} \alpha_{j} \delta\left(\cdot-x_{j}\right), \quad m \in \mathbb{N}
$$

for any fixed set $\alpha:=\left\{\alpha_{j}\right\}_{j=1}^{m} \subset \mathbb{R}$. The first one is based on the quadratic forms method. Another way to introduce local interactions on $X:=\left\{x_{j}\right\}_{j=1}^{m} \subset \mathbb{R}$ is to consider maximal operator corresponding to $\mathfrak{L}_{1}$ and impose boundary conditions at $x_{j}, \quad j \in\{1, \ldots, m\}$ (see [3]), i.e.,

$$
\operatorname{dom}\left(H_{X, \alpha}\right)=\left\{f \in W^{2,2}(\mathbb{R} \backslash X) \cap W^{1,2}(\mathbb{R}): f^{\prime}\left(x_{j}+\right)-f^{\prime}\left(x_{j}-\right)=\alpha_{j} f\left(x_{j}\right)\right\} .
$$

In contrast to one-dimensional case, the differential expression (1.1) does not define an operator in $L^{2}\left(\mathbb{R}^{d}\right)$, $d \geq 2$, by means of the quadratic forms since the linear functional $\delta_{x}: f \rightarrow f(x)$ is not continuous in $W^{1,2}\left(\mathbb{R}^{d}\right)$ for $d \geq 2$. However, it is still possible to apply the extension theory approach. Namely, F. Berezin and L. Faddeev in their pioneering paper [8] proposed to consider 1.1] (with $m=1$ and $d=3$ ) in the framework of extension theory. They associated with $\mathfrak{L}_{d}$ the family of all self-adjoint extensions of the following symmetric operator

$$
H:=-\Delta, \quad \operatorname{dom}(H):=\left\{f \in W^{2,2}\left(\mathbb{R}^{d}\right): f\left(x_{j}\right)=0, \quad j \in\{1, . ., m\}\right\}, m \in \mathbb{N} .
$$

\footnotetext{
* Corresponding author E-mail: nataliia@ime.usp.br,

** E-mail: mmm@telenet.dn.ua.

*** E-mail: zastavn@rambler.ru.
} 
It is well known that $H$ is closed non-negative symmetric operator with equal deficiency indices $n_{ \pm}(H)=m$ (see [3]). In [3], the authors proposed to associate with the Hamiltonian (1.1) certain $m$-parametric family $H_{X, \alpha}^{(d)}$ describing local point interactions. They parameterized the family in terms of the resolvents. The latter enabled the authors to obtain an explicit description of the spectrum for any operator from the family $H_{X, \alpha}^{(d)}$.

In the recent publications [5, 10,20], boundary triplets and the corresponding Weyl functions technique (see [12,19] and also Section 2.1) was involved to investigate multi-dimensional Schrödinger operators with point interactions (the cases $d \in\{2,3\}$ ). In the present paper, we apply boundary triplets approach to parametrize all self-adjoint extensions of $H$. Besides, using Weyl functions technique, we investigate their spectra. Moreover, we substantially involve the theory of radial positive definite functions [2, chapter V] in our approach. In particular, we employ strict positive definiteness (see Definition 3.1) of the functions $\frac{\sin s|\cdot|}{s|\cdot|}$ and the Bessel functions $J_{0}(s|\cdot|)$ with any $s>0$ on $\mathbb{R}^{3}$ and $\mathbb{R}^{2}$, respectively, to prove pure absolute continuity of non-negative spectrum of any self-adjoint realization of $\mathfrak{L}_{d}$. For this purpose we complete the classical Schoenberg theorem [31] regarding the integral representation of radial positive definite functions.

The paper is organized as follows. Section 2 is introductory. It contains definitions of a boundary triplet and the corresponding Weyl function [12, 19] and also facts about the Weyl functions [9, 23]. In Section 3, we complete Schoenberg theorem by establishing strict positive definiteness of any non-constant radial positive definite function on $\mathbb{R}^{n}, n \geq 2$. In Sections 4 and 5, we investigate 3D- and 2D-Schrödinger operators with point interactions, respectively.

Namely, in Subsection 4.1 (resp., 5.1), we define boundary triplet $\Pi=\left\{\mathcal{H}, \Gamma_{0}, \Gamma_{1}\right\}$ for $H^{*}$ and compute the corresponding Weyl function. It appears to be close to that contained in Krein's resolvent formula for the family $H_{X, \alpha}^{(d)}$ in [3]. In particular, for the proof of the surjectivity of the mapping $\Gamma=\left(\Gamma_{0}, \Gamma_{1}\right)^{\top}$ we employ the strict positive definiteness of the function $e^{-|\cdot|}$ on $\mathbb{R}^{n}$ for any $n \in \mathbb{N}$.

Subsections 4.2 and 5.2 are devoted to the spectral analysis of the self-adjoint realizations of $\mathfrak{L}_{d}$. To investigate the absolutely continuous spectrum we apply technique elaborated in [9,23]. For this purpose we need invertibility of the matrices

$$
\left(\delta_{k j}+\frac{\sin \left(\sqrt{x}\left|x_{k}-x_{j}\right|\right)}{\sqrt{x}\left|x_{k}-x_{j}\right|+\delta_{k j}}\right)_{j, k=1}^{m} \quad \text { and } \quad\left(J_{0}\left(\sqrt{x}\left|x_{j}-x_{k}\right|\right)\right)_{j, k=1}^{m}, \quad x \in \mathbb{R}_{+},
$$

which we extract from the strict positive definiteness of the functions $\frac{\sin s|\cdot|}{s|\cdot|}$ and $J_{0}(s|\cdot|), s>0$, on $\mathbb{R}^{3}$ and $\mathbb{R}^{2}$, respectively. We emphasize that in the proof of Theorems 4.7 and 5.7 our complement to Schoenberg theorem is used in full generality. Indeed, it follows from the integral representation (3.2) that the strict positive definiteness of $\frac{\sin s|\cdot|}{s|\cdot|}$ and $J_{0}(s|\cdot|)$ for all $s>0$ yields the strict positive definiteness of any radial positive definite function $f$ on $\mathbb{R}^{3}$ and $\mathbb{R}^{2}$, respectively.

Finally, description of the non-negative self-adjoint extensions of $H(d=3)$ is provided in Subsection 4.3. For suitable choice of a boundary triplet $\widetilde{\Pi}$ strong resolvent limit of the corresponding Weyl function $\widetilde{M}(x)$ at $x=0$ appears to be positive definite matrix in a view of strict positive definiteness of the function $\frac{1-e^{-|\cdot|}}{|\cdot|}$ on $\mathbb{R}^{n}$ for any $n \in \mathbb{N}$.

Notation. Let $\mathfrak{H}$ and $\mathcal{H}$ denote separable Hilbert spaces; $[\mathfrak{H}, \mathcal{H}]$ denotes the space of bounded linear operators from $\mathfrak{H}$ to $\mathcal{H},[\mathcal{H}]:=[\mathcal{H}, \mathcal{H}]$; the set of closed operators in $\mathcal{H}$ is denoted by $\mathcal{C}(\mathcal{H})$. Let $A$ be a linear operator in a Hilbert space $\mathfrak{H}$. In what follows domain, kernel, and range of $A$ are denoted by $\operatorname{dom}(A), \operatorname{ker}(A), \operatorname{ran}(A)$, respectively; $\sigma(A)$ and $\rho(A)$ denote the spectrum and the resolvent set of $A ; \mathfrak{N}_{z}$ denotes the defect subspace of $A ; C[0, \infty)$ denotes the Banach space of functions continuous and bounded on $[0, \infty)$.

\section{Extension theory of symmetric operators}

\subsection{Boundary triplets and proper extensions}

In this subsection, we recall basic notions and facts of the theory of boundary triplets (we refer the reader to [12,13,19] for a detailed exposition). In what follows $A$ always denotes a closed symmetric operator in separable Hilbert space $\mathfrak{H}$ with equal deficiency indices $n_{-}(A)=n_{+}(A) \leq \infty$. 
Definition 2.1 [19] A totality $\Pi=\left\{\mathcal{H}, \Gamma_{0}, \Gamma_{1}\right\}$ is called a boundary triplet for the adjoint operator $A^{*}$ of $A$ if $\mathcal{H}$ is an auxiliary Hilbert space and $\Gamma_{0}, \Gamma_{1}: \operatorname{dom}\left(A^{*}\right) \rightarrow \mathcal{H}$ are linear mappings such that

(i) the following abstract second Green identity holds

$$
\left(A^{*} f, g\right)_{\mathfrak{H}}-\left(f, A^{*} g\right)_{\mathfrak{H}}=\left(\Gamma_{1} f, \Gamma_{0} g\right)_{\mathcal{H}}-\left(\Gamma_{0} f, \Gamma_{1} g\right)_{\mathcal{H}}, \quad f, g \in \operatorname{dom}\left(A^{*}\right)
$$

(ii) the mapping $\Gamma:=\left(\Gamma_{0}, \Gamma_{1}\right)^{\top}: \operatorname{dom}\left(A^{*}\right) \rightarrow \mathcal{H} \oplus \mathcal{H}$ is surjective.

With a boundary triplet $\Pi=\left\{\mathcal{H}, \Gamma_{0}, \Gamma_{1}\right\}$ for $A^{*}$ one associates two self-adjoint extensions of $A$ defined by

$$
A_{0}:=A^{*} \uparrow \operatorname{ker}\left(\Gamma_{0}\right) \quad \text { and } \quad A_{1}:=A^{*} \uparrow \operatorname{ker}\left(\Gamma_{1}\right)
$$

Definition 2.2 $(i)$ A closed extension $\widetilde{A}$ of $A$ is called proper if $A \subseteq \widetilde{A} \subseteq A^{*}$. The set of all proper extensions of $A$ is denoted by $\operatorname{Ext}_{A}$.

(ii) Two proper extensions $\widetilde{A}_{1}$ and $\widetilde{A}_{2}$ of $A$ are called disjoint if $\operatorname{dom}\left(\widetilde{A}_{1}\right) \cap \operatorname{dom}\left(\widetilde{A}_{2}\right)=\operatorname{dom}(A)$.

Remark 2.3 $(i)$ For any symmetric operator $A$ with $n_{+}(A)=n_{-}(A)$, a boundary triplet $\Pi=\left\{\mathcal{H}, \Gamma_{0}, \Gamma_{1}\right\}$ for $A^{*}$ exists and is not unique [19]. It is known also that $\operatorname{dim} \mathcal{H}=n_{ \pm}(A)$ and $\operatorname{ker} \Gamma=\operatorname{ker}\left(\Gamma_{0}, \Gamma_{1}\right)^{\top}=\operatorname{dom}(A)$.

(ii) Moreover, for each self-adjoint extension $\widetilde{A}$ of $A$ there exists a boundary triplet $\Pi=\left\{\mathcal{H}, \Gamma_{0}, \Gamma_{1}\right\}$ such that $\widetilde{A}=A^{*} \uparrow \operatorname{ker}\left(\Gamma_{0}\right)=: A_{0}$.

(iii) For each boundary triplet $\Pi=\left\{\mathcal{H}, \Gamma_{0}, \Gamma_{1}\right\}$ for $A^{*}$ and each bounded self-adjoint operator $B$ in $\mathcal{H}$ a triplet $\Pi_{B}=\left\{\mathcal{H}, \Gamma_{0}^{B}, \Gamma_{1}^{B}\right\}$ with $\Gamma_{1}^{B}:=\Gamma_{0}$ and $\Gamma_{0}^{B}:=B \Gamma_{0}-\Gamma_{1}$ is also a boundary triplet for $A^{*}$.

A role of a boundary triplet for $A^{*}$ in the extension theory is similar to that of coordinate system in the analytic geometry. Namely, it allows one to parameterize the set Ext ${ }_{A}$ by means of linear relations in $\mathcal{H}$ in place of $\mathrm{J}$.von Neumann formulas. To explain this we recall the following definitions.

Definition $2.4(i)$ A closed linear relation $\Theta$ in $\mathcal{H}$ is a closed subspace of $\mathcal{H} \oplus \mathcal{H}$.

(ii) A linear relation $\Theta$ is symmetric if $\left(g_{1}, f_{2}\right)-\left(f_{1}, g_{2}\right)=0$ for all $\left\{f_{1}, g_{1}\right\},\left\{f_{2}, g_{2}\right\} \in \Theta$.

(iii) The adjoint relation $\Theta^{*}$ is defined by

$$
\Theta^{*}=\left\{\left\{k, k^{\prime}\right\}:\left(h^{\prime}, k\right)=\left(h, k^{\prime}\right) \text { for all }\left\{h, h^{\prime}\right\} \in \Theta\right\} .
$$

(iv) A closed linear relation $\Theta$ is called self-adjoint if both $\Theta$ and $\Theta^{*}$ are maximal symmetric, i.e., they do not admit symmetric extensions.

For the symmetric relation $\Theta \subseteq \Theta^{*}$ in $\mathcal{H}$ the multivalued part mul $(\Theta)$ is the orthogonal complement of $\operatorname{dom}(\Theta)$ in $\mathcal{H}$. Setting $\mathcal{H}_{\mathrm{op}}:=\overline{\operatorname{dom}(\Theta)}$ and $\mathcal{H}_{\infty}=\operatorname{mul}(\Theta)$, one verifies that $\Theta$ can be rewritten as the direct orthogonal sum of a self-adjoint operator $\Theta_{\text {op }}$ in the subspace $\mathcal{H}_{\text {op }}$ and a "pure" relation $\Theta_{\infty}=\left\{\left\{0, f^{\prime}\right\}: f^{\prime} \in\right.$ $\operatorname{mul}(\Theta)\}$ in the subspace $\mathcal{H}_{\infty}$.

Proposition 2.5 12, Let $\Pi=\left\{\mathcal{H}, \Gamma_{0}, \Gamma_{1}\right\}$ be a boundary triplet for $A^{*}$. Then the mapping

$$
\operatorname{Ext}_{A} \ni \widetilde{A}:=A_{\Theta} \rightarrow \Theta:=\Gamma(\operatorname{dom}(\widetilde{A}))=\left\{\left\{\Gamma_{0} f, \Gamma_{1} f\right\}: f \in \operatorname{dom}(\widetilde{A})\right\}
$$

establishes a bijective correspondence between the set of all closed proper extensions Ext ${ }_{A}$ of $A$ and the set of all closed linear relations $\widetilde{\mathcal{C}}(\mathcal{H})$ in $\mathcal{H}$. Furthermore, the following assertions hold.

(i) The equality $\left(A_{\Theta}\right)^{*}=A_{\Theta}$ holds for any $\Theta \in \widetilde{\mathcal{C}}(\mathcal{H})$.

(ii) The extension $A_{\Theta}$ in (2.2) is symmetric (self-adjoint) if and only if $\Theta$ is symmetric (self-adjoint). Moreover, $n_{ \pm}\left(A_{\Theta}\right)=n_{ \pm}(\Theta)$.

(iii) If, in addition, the closed extensions $A_{\Theta}$ and $A_{0}$ are disjoint, then (2.2) takes the form

$$
A_{\Theta}=A_{B}=A^{*} \uparrow \operatorname{dom}\left(A_{B}\right), \quad \operatorname{dom}\left(A_{B}\right)=\operatorname{dom}\left(A^{*}\right) \uparrow \operatorname{ker}\left(\Gamma_{1}-B \Gamma_{0}\right), \quad B \in \mathcal{C}(\mathcal{H}) .
$$

\subsection{Weyl function, $\gamma$-field and spectra of proper extensions}

It is known that Weyl function is an important tool in the spectral theory of singular Sturm-Liouville operators. In [12,13] the concept of Weyl function was generalized to an arbitrary symmetric operator $A$ with equal deficiency indices. In this subsection we recall basic facts about Weyl functions. 
Definition 2.6 [12] Let $\Pi=\left\{\mathcal{H}, \Gamma_{0}, \Gamma_{1}\right\}$ be a boundary triplet for $A^{*}$. The operator valued function $M(\cdot)$ : $\rho\left(A_{0}\right) \rightarrow[\mathcal{H}]$ defined by

$$
M(z) \Gamma_{0} f_{z}=\Gamma_{1} f_{z}, \quad f_{z} \in \mathfrak{N}_{z}, z \in \rho\left(A_{0}\right),
$$

is called the Weyl function, corresponding to the triplet $\Pi$.

The definition of the Weyl function is correct and the Weyl function $M(\cdot)$ is Nevanlinna or R-function.

In the following we will be concerned with a simple symmetric operators. Recall that a symmetric operator $A$ is said to be simple if there is no nontrivial subspace which reduces it to self-adjoint operator.

The spectrum and the resolvent set of the closed (not necessarily self-adjoint) extensions of simple symmetric operator $A$ can be described with the help of the function $M(\cdot)$. Namely, the following proposition holds.

Proposition 2.7 Let $A$ be a densely defined simple symmetric operator in $\mathfrak{H}, \Theta \in \widetilde{\mathcal{C}}(\mathcal{H}), A_{\Theta} \in \operatorname{Ext}_{A}$, and $z \in \rho\left(A_{0}\right)$. Then the following equivalences hold.

(i) $z \in \rho\left(A_{\Theta}\right) \Longleftrightarrow 0 \in \rho(\Theta-M(z))$;

(ii) $z \in \sigma_{\tau}\left(A_{\Theta}\right) \Longleftrightarrow 0 \in \sigma_{\tau}(\Theta-M(z)), \quad \tau \in\{p, c, r\}$;

(iii) $f_{z} \in \operatorname{ker}\left(A_{\Theta}-z\right) \quad \Longleftrightarrow \quad \Gamma_{0} f_{z} \in \operatorname{ker}(\Theta-M(z)) \quad$ and $\operatorname{dim} \operatorname{ker}\left(A_{\Theta}-z\right)=\operatorname{dim} \operatorname{ker}(\Theta-M(z))$.

The following proposition gives us quantitative characterization of the negative spectrum of self-adjoint extensions of the operator $A$.

Proposition 2.8 [12] Let $A$ be a densely defined non-negative symmetric operator in $\mathfrak{H}$, and let $\Pi=$ $\left\{\mathcal{H}, \Gamma_{0}, \Gamma_{1}\right\}$ be a boundary triplet for $A^{*}$. Let also $M(\cdot)$ be the corresponding Weyl function and $A_{0}=A_{F}$, where $A_{F}$ is the Friedrichs extension of $A$. Then the following assertions hold.

(i) The strong resolvent limit $M(0):=s-R-\lim _{x \uparrow 0} M(x)$ exists and is self-adjoint linear relation semibounded from below.

(ii) If, in addition, $M(0) \in[\mathcal{H}]$, then the number of negative squares of $A_{\Theta}=A_{\Theta}^{*}$ equals the number of negative squares of the relation $\Theta-M(0)$, i.e., $\kappa_{-}\left(A_{\Theta}\right)=\kappa_{-}(\Theta-M(0))$.

In particular, the self-adjoint extension $A_{\Theta}$ of $A$ is non-negative if and only if the linear relation $\Theta-M(0)$ is non-negative.

Denote

$$
\begin{aligned}
& M(x+i 0):=s-\lim _{y \downarrow 0} M(x+i y), \quad d_{M}(x):=\operatorname{rank}(\operatorname{Im}(M(x+i 0))), \\
& M_{h}(z):=(M(z) h, h), \Omega_{a c}\left(M_{h}\right):=\left\{x \in \mathbb{R}: 0<\operatorname{Im}\left(M_{h}(x+i 0)\right)<+\infty\right\}, z \in \mathbb{C}_{+}, h \in \mathcal{H},
\end{aligned}
$$

where $M_{h}(x+i 0):=\lim _{y \downarrow 0}(M(x+i y) h, h)$. Since $\operatorname{Im}\left(M_{h}(z)\right)>0, z \in \mathbb{C}_{+}$, the limit $M_{h}(x+i 0)$ exists and is finite for a.e. $x \in \mathbb{R}$.

To state the next proposition we need a concept of the absolutely continuous closure $\mathrm{cl}_{a c}(\delta)$ of a Borel subset $\delta \subset \mathbb{R}$ introduced in [9] and [16]. We refer to [16,23] for the definition and basic properties.

Proposition 2.9 [9]23] Let A be a simple densely defined closed symmetric operator with equal deficiency indices in separable Hilbert space $\mathfrak{H}$. Let $\Pi=\left\{\mathcal{H}, \Gamma_{0}, \Gamma_{1}\right\}$ be a boundary triplet for $A^{*}$ and $M(\cdot)$ the corresponding Weyl function. Assume also that $\tau=\left\{h_{k}\right\}_{k=1}^{N}, \quad 1 \leq N \leq \infty$ is a total set in $\mathcal{H}$. Let also $A_{B}=A^{*}\left\lceil\operatorname{ker}\left(\Gamma_{1}-B \Gamma_{0}\right)\right.$, with $B=B^{*} \in \mathcal{C}(\mathcal{H})$. Then the following assertions hold.

(i) The operator $A_{0}$ has no singular continuous spectrum within the interval $(a, b)$ iffor each $k \in\{1,2, . ., N\}$ the set $(a, b) \backslash \Omega_{a c}\left(M_{h_{k}}\right)$ is countable.

(ii) If the limit $M(x+i 0)$ exists for a.e. $x \in \mathbb{R}$, then $\sigma_{a c}\left(A_{0}\right)=\mathrm{cl}_{a c}\left(\operatorname{supp}\left(d_{M}(x)\right)\right)$.

(iii) For any Borel subset $\mathcal{D} \subset \mathbb{R}$ the absolutely continuous parts $A_{0} E_{A_{0}}^{a c}(\mathcal{D})$ and $A_{B} E_{A_{B}}^{a c}(\mathcal{D})$ of the operators $A_{0} E_{A_{0}}(\mathcal{D})$ and $A_{B} E_{A_{B}}(\mathcal{D})$ are unitarily equivalent if and only if $d_{M}(x)=d_{M_{B}}(x)$ for a.e. $x \in \mathcal{D}$.

\section{Positive definite functions. Complement of the Schoenberg theorem}

Let $(u, v)=u_{1} v_{1}+\ldots+u_{n} v_{n}$ be a scalar product of two vectors $u=\left(u_{1}, \ldots, u_{n}\right)$ and $v=\left(v_{1}, \ldots, v_{n}\right)$ from $\mathbb{R}^{n}, n \in \mathbb{N}$, and let $|u|=\sqrt{(u, u)}$ be Euclidean norm. Recall some basic facts and notions of the theory of positive definite functions [2]. 
Definition 3.1 [2, 33] $(i)$ Function $g(\cdot): \mathbb{R}^{n} \rightarrow \mathbb{C}$ is said to be positive definite on $\mathbb{R}^{n}$ and is referred to the class $\Phi\left(\mathbb{R}^{n}\right)$ if it is continuous at 0 and for any finite subsets $X:=\left\{x_{k}\right\}_{k=1}^{m} \subset \mathbb{R}^{n}$ and $\xi:=\left\{\xi_{k}\right\}_{k=1}^{m} \subset \mathbb{C}, m \in$ $\mathbb{N}$ the following inequality holds

$$
\sum_{k, j=1}^{m} \xi_{k} \bar{\xi}_{j} g\left(x_{k}-x_{j}\right) \geq 0
$$

(ii) Moreover, $g(\cdot)$ is said to be strictly positive on $\mathbb{R}^{n}$ if the inequality 3.1 is strict for any subset of distinct points $X=\left\{x_{k}\right\}_{k=1}^{m} \subset \mathbb{R}^{n}$ and for any subset $\xi=\left\{\xi_{k}\right\}_{k=1}^{m} \subset \mathbb{C}$ satisfying condition $\sum_{k=1}^{m}\left|\xi_{k}\right|>0$.

Clearly, positive definiteness of the function $g(\cdot)$ is equivalent to the non-negative definiteness of the matrix $G(X)=\left(g_{k j}\right)_{k, j=1}^{m}$ with $g_{k j}=g\left(x_{k}-x_{j}\right)$ for any subset $X=\left\{x_{k}\right\}_{k=1}^{m} \subset \mathbb{R}^{n}$, while its strict positive definiteness is equivalent to (strict) positive definiteness of the matrix $G(X)$ for any subset of distinct points $X=\left\{x_{k}\right\}_{k=1}^{m} \subset \mathbb{R}^{n}$.

The following classical Bochner theorem gives the description of the class $\Phi\left(\mathbb{R}^{n}\right)$.

Theorem 3.2 [11] A function $g(\cdot)$ is positive definite on $\mathbb{R}^{n}$ if and only if

$$
g(x)=\int_{\mathbb{R}^{n}} e^{i(u, x)} d \mu(u),
$$

where $\mu$ is a finite non-negative Borel measure on $\mathbb{R}^{n}$.

Definition 3.3 A function $f(\cdot) \in C[0,+\infty)$ is said to be radial positive definite function of the class $\Phi_{n}$, $n \in \mathbb{N}$, if $f(|\cdot|)$ is positive definite on $\mathbb{R}^{n}$, i.e., if $f(|\cdot|) \in \Phi\left(\mathbb{R}^{n}\right)$.

A characterization of the class $\Phi_{n}$ is given by the following Schoenberg theorem [30,31] (see also [2, Theorem 5.4.2]).

Theorem 3.4 Function $f(\cdot)$ belongs to the class $\Phi_{n}$ if and only if

$$
f(t)=\int_{0}^{+\infty} \Omega_{n}(s t) d \mu(s), \quad t \geq 0,
$$

where $\mu$ is a non-negative finite Borel measure on $[0, \infty)$, and

$$
\begin{aligned}
& \Omega_{n}(t)=\Gamma\left(\frac{n}{2}\right)\left(\frac{2}{t}\right)^{\frac{n-2}{2}} J_{\frac{n-2}{2}}(t)=\sum_{p=0}^{\infty}\left(-\frac{t^{2}}{4}\right)^{p} \frac{\Gamma\left(\frac{n}{2}\right)}{p ! \Gamma\left(\frac{n}{2}+p\right)}, \\
& \Omega_{n}(|x|)=\int_{S_{n}} e^{i(u, x)} d \nu_{n}(u), x \in \mathbb{R}^{n} .
\end{aligned}
$$

Here $\nu_{n}$ is the Borel measure uniformly distributed over the unit sphere $S_{n}$ centered at the origin and $\nu_{n}\left(S_{n}\right)=1$.

Remark 3.5 It is not difficult to show that for any $n \in \mathbb{N}$ the strict inclusion $\Phi_{n+1} \subset \Phi_{n}$ takes place. Indeed, it is known [18], [34, Theorem 5], [35, Example 1] that $(1-|t|)_{+}^{\delta} \in \Phi_{n}$ if and only if $\delta \geq \frac{n+1}{2}$. Earlier Askey [6] and Trigub [32] considered the case of natural $\delta$ and proved the necessity for odd $n$.

On the other hand, the strict inclusion $\Phi_{n+1} \subset \Phi_{n}$ follows from another example. Namely, $\operatorname{Re}\left(e^{-z t}\right) \in \Phi_{n}$ if and only if $|\arg z| \leq \pi /(2 n), z \in \mathbb{C}$ (see [34. Theorem 3]). Therefore $\Omega_{n} \in \Phi_{n}$, but $\Omega_{n} \notin \Phi_{n+1}, n \in \mathbb{N}$.

Our complement to the Schoenberg theorem reads as follows.

Theorem 3.6 If $f(\cdot) \in \Phi_{n}, n \geq 2$, and $f(\cdot) \not \equiv$ const on $[0,+\infty)$, then the function $f(|\cdot|)$ is strictly positive definite on $\mathbb{R}^{n}$.

We start with the following auxiliary lemma and present two different proofs.

Lemma 3.7 Let $S_{n}^{r}(y)$ be a sphere of radius $r$ in $\mathbb{R}^{n}$ centered at $y$ and $n \geq 2$, let also $X=\left\{x_{k}\right\}_{k=1}^{m}$ be a subset of $\mathbb{R}^{n}$ such that $x_{p} \neq x_{j}$ as $p \neq j$ and $\xi=\left\{\xi_{k}\right\}_{k=1}^{m} \subset \mathbb{C}$. If

$$
\sum_{p=1}^{m} \xi_{p} e^{i\left(u, x_{p}\right)}=0, \quad \text { for all } u \in S_{n}^{r}(y),
$$

then $\xi_{p}=0$ for $p \in\{1, . ., m\}$. 
The first proof. Without loss of generality we may assume that $y=0$ and $r=1$. Let also for definiteness $\xi_{1} \neq 0$. For $m=1$ the statement is obvious. Put $m \geq 2$. Denote $\max _{1 \leq p \leq m}\left|x_{p}\right|=R_{0}>0$. Let $\left\{e_{j}\right\}_{j=1}^{n}$ be the standard orthogonal basis in $\mathbb{R}^{n}$. We may assume that $x_{1}=R_{0} e_{1}$. Let $P$ be the orthogonal projector onto $\operatorname{span}\left\{e_{1}, e_{2}\right\}$. Then $P x_{p}=r_{p}\left(\cos \varphi_{p} e_{1}+\sin \varphi_{p} e_{2}\right)$ with $0 \leq r_{p}=\left|P x_{p}\right| \leq\left|x_{p}\right| \leq R_{0}, \quad 0 \leq \varphi_{p}<2 \pi$. Assume that $r_{1}=r_{2}=\ldots=r_{m^{\prime}}=R_{0}$ and $r_{p}<R_{0}$ as $p>m^{\prime}$ (if $m^{\prime}<m$ ). Then $P x_{p}=x_{p}, 1 \leq p \leq m^{\prime}$. Evidently, we may also assume that $0=\varphi_{1}<\varphi_{2}<\ldots<\varphi_{m^{\prime}}<2 \pi$. Put in (3.5) $u=\cos \varphi e_{1}+\sin \varphi e_{2} \in$ $S_{n}^{1}(0), \varphi \in \mathbb{R}$. Therefore the equality 3.5 takes the form

$$
\sum_{p=1}^{m} \xi_{p} \exp \left(i r_{p} \cos \left(\varphi-\varphi_{p}\right)\right)=0, \quad \varphi \in \mathbb{R}
$$

It is well known that generating function for the Bessel functions admits the following representation [15 chapter $19, \S 3]$

$$
e^{\frac{a}{2}\left(z-z^{-1}\right)}=\sum_{k=-\infty}^{\infty} J_{k}(a) z^{k}, \quad z \neq 0, \quad a \in \mathbb{C} .
$$

Putting in (3.7) $z=i e^{i\left(\varphi-\varphi_{p}\right)}$ and $a=r_{p}, \quad p \in\{1, . ., m\}$, we arrive at the following expansion into Fourier series for the functions $f_{p}(\varphi)=\exp \left(i r_{p} \cos \left(\varphi-\varphi_{p}\right)\right)$

$$
\exp \left(i r_{p} \cos \left(\varphi-\varphi_{p}\right)\right)=\sum_{k=-\infty}^{\infty} J\left(r_{p}\right) i^{k} e^{-i k \varphi_{p}} e^{i k \varphi}
$$

It is easily seen that from (3.6) and (3.8) follows the equality

$$
\sum_{k=-\infty}^{\infty}\left[\sum_{p=1}^{m} \xi_{p} \exp \left(-i k \varphi_{p}\right) J_{k}\left(r_{p}\right) i^{k}\right] e^{i k \varphi}=0 .
$$

Therefore

$$
\sum_{p=1}^{m} \xi_{p} \exp \left(-i k \varphi_{p}\right) J_{k}\left(r_{p}\right)=0, \quad k \in \mathbb{N} .
$$

Using the following expansion into series for $J_{k}(x)$ ( [27, Section 2])

$$
J_{k}(x)=\left(\frac{x}{2}\right)^{k} \sum_{p=0}^{\infty}\left(-\frac{x^{2}}{4}\right)^{p} \frac{1}{p ! \Gamma(k+p+1)},
$$

we get $k ! 2^{k} J_{k}(x)=x^{k}\left[1+a_{k}(x)\right]$, where

$$
\left|a_{k}(x)\right| \leq(k+1)^{-1}\left[\exp \left(\frac{x^{2}}{4}\right)-1\right], \quad x \in \mathbb{R}, k \in \mathbb{N} .
$$

Multiplying the equality (3.9) by $k ! 2^{k} R_{0}^{-k}$, we obtain

$$
\sum_{p=1}^{m^{\prime}} \xi_{p} \exp \left(-i k \varphi_{p}\right)\left[1+a_{k}\left(R_{0}\right)\right]=-\sum_{p=m^{\prime}+1}^{m} \xi_{p} \exp \left(-i k \varphi_{p}\right)\left(r_{p} R_{0}^{-1}\right)^{k}\left[1+a_{k}\left(r_{p}\right)\right]
$$

where right-hand side equals 0 if $m^{\prime}=m$. If $m^{\prime}<m$, then $r_{p} R_{0}^{-1}<1$ as $p>m^{\prime}$. Thereby,

$$
\lim _{k \rightarrow \infty} \sum_{p=1}^{m^{\prime}} \xi_{p} \exp \left(-i k \varphi_{p}\right)=0
$$


Since arithmetic means of the sequence in (3.10) converges to $\xi_{1}\left(\varphi_{1}=0\right)$, then $\xi_{1}=0$. Thus, the theorem is proved.

The second proof. As in the first proof, we reduce considerations to investigation of equality (3.6). By uniqueness theorem for analytic functions, equality (3.6) remains valid for any $z \in \mathbb{C}$

$$
\sum_{p=1}^{m} \xi_{p} \exp \left(i r_{p} \cos \left(z-\varphi_{p}\right)\right)=0, \quad z=x+i y \in \mathbb{C} .
$$

Since, by Euler formula, $\cos \left(z-\varphi_{p}\right)=\left(e^{i\left(z-\varphi_{p}\right)}+e^{-i\left(z-\varphi_{p}\right)}\right) / 2$, we have

$$
\left|\exp \left(i r_{p} \cos \left(z-\varphi_{p}\right)\right)\right|=\exp \left(-\frac{r_{p}}{2} \operatorname{Im}\left(e^{i\left(z-\varphi_{p}\right)}+e^{-i\left(z-\varphi_{p}\right)}\right)\right)=\exp \left(r_{p} \operatorname{sh} y \sin \left(x-\varphi_{p}\right)\right) .
$$

Denote $\psi_{p}(z)=\arg \left(\exp \left(i r_{p} \cos \left(z-\varphi_{p}\right)\right)\right) \in[0,2 \pi)$. Thus, by $\underline{3.11}$,

$$
\sum_{p=1}^{m} \xi_{p}\left[\exp \left(r_{p} \operatorname{sh} y \sin \left(x-\varphi_{p}\right)\right)\right] e^{i \psi_{p}(z)}=0
$$

Multiplying (3.12) by $\exp \left(-R_{0} \operatorname{sh} y\right)$, we arrive at

$$
\sum_{p=1}^{m^{\prime}} \xi_{p}\left[\exp \left(R_{0} \operatorname{sh} y\left(\sin \left(x-\varphi_{p}\right)-1\right)\right)\right] e^{i \psi_{p}(z)}+\sum_{p=m^{\prime}+1}^{m} \xi_{p}\left[\exp \left(\operatorname{sh} y\left(r_{p} \sin \left(x-\varphi_{p}\right)-R_{0}\right)\right)\right] e^{i \psi_{p}(z)}=0 .
$$

Setting $x=\varphi_{1}+\frac{\pi}{2}$ in (3.13) and passing to the limit as $y \rightarrow+\infty$, we obtain $\xi_{1}=0$.

Remark 3.8 The first proof of Lemma 3.7 belongs to Viktor Zastavnyi. Chronologically it was obtained earlier then the second proof proposed by the other two authors.

Remark 3.9 It might be easily shown that Lemma3.7 is not valid for any manifold in $\mathbb{R}^{n}$. Below we give the explanatory example. It is obvious that any hyperplane $\pi_{a}$ in $\mathbb{R}^{n}, n \geq 2$, which does not contain the origin, is given by

$$
\pi_{a}=\left\{u \in \mathbb{R}^{n}:(u, a)=1, \quad \text { where } a \in \mathbb{R}^{n}, a \neq 0\right\} .
$$

Then on such hyperplane the expression $1+\exp (i(u, \pi a))$ is identically zero. Thus, for any finite set of hyperplanes with the above property there exists a set of points $y_{k} \in \mathbb{R}^{n}, y_{k} \neq 0, k \in\{1, . ., q\}$ such that

$$
0 \equiv \prod_{k=1}^{q}\left(1+e^{i\left(u, \pi y_{k}\right)}\right)=\sum_{p=1}^{m} \xi_{p} e^{i\left(u, x_{p}\right)}, \quad u \in \bigcup_{k=1}^{q} \pi_{y_{k}}
$$

Here $m \geq 2, \xi_{p}>0, p \in\{1, . ., m\}$ and $X=\left\{x_{p}\right\}_{p=1}^{m}$ is a subset of $\mathbb{R}^{n}$ such that $x_{p} \neq x_{j}$ as $p \neq j$.

Now we are ready to prove the complement of Theorem 3.4 Below we present two slightly different proofs.

The first proof of Theorem 3.6 Let $\mu$ be non-negative finite Borel measure on $[0,+\infty)$ from the representation (3.2) for the function $f$. It is obvious that $\mu((0,+\infty))>0$ (otherwise, $f(t) \equiv f(0)$ ).

Let $X=\left\{x_{k}\right\}_{k=1}^{m} \subset \mathbb{R}^{n}$ and $\xi=\left\{\xi_{k}\right\}_{k=1}^{m} \subset \mathbb{C}$ be subsets such that $x_{p} \neq x_{j}$ as $p \neq j$ and $\sum_{k=1}^{m}\left|\xi_{k}\right|>0$. From Lemma 3.7 with $y=0, r=1$ it follows that

$$
\int_{S_{n}}\left|\sum_{k=1}^{m} \xi_{k} e^{i\left(u, s x_{k}\right)}\right|^{2} d \nu_{n}(u)>0 \text { for any } s>0,
$$


where $\nu_{n}$ is the measure from representation (3.4). Since $\mu((0,+\infty))>0$, we get

$$
\sum_{k, j=1}^{m} \xi_{k} \bar{\xi}_{j} f\left(\left|x_{k}-x_{j}\right|\right)=\int_{0}^{+\infty}\left(\int_{S_{n}}\left|\sum_{k=1}^{m} \xi_{k} e^{i\left(u, s x_{k}\right)}\right|^{2} d \nu_{n}(u)\right) d \mu(s)>0 .
$$

Thus, the theorem is proved.

The second proof. By Theorem 3.2, we have

$$
f(|x|)=\int_{\mathbb{R}^{n}} e^{i(u, x)} d \mu(u), \quad x \in \mathbb{R}^{n},
$$

where $\mu$ is non-negative finite Borel measure on $\mathbb{R}^{n}$. It is easily seen that $\operatorname{supp} \mu$ is a radial set, i.e., if $x_{0} \in$ $\operatorname{supp} \mu$, then the support contains sphere $S_{n}^{r}(0)$ of radius $r=\left|x_{0}\right| \geq 0$ centered at the origin. If $f(t) \not \equiv f(0)$, then $\operatorname{supp} \mu$ contains a sphere $S_{n}^{r}(0)$.

Let $f(t) \not \equiv$ const, $m \in \mathbb{N}$, and the set $X=\left\{x_{k}\right\}_{k=1}^{m} \subset \mathbb{R}^{n}$ is such that $x_{k} \neq x_{j}$ as $k \neq j$. Consider the following quadratic form in $\mathbb{C}^{m}$

$$
Q(\xi):=\sum_{k, j=1}^{m} \xi_{k} \bar{\xi}_{j} f\left(\left|x_{k}-x_{j}\right|\right)=\int_{\mathbb{R}^{n}}\left|\sum_{k=1}^{m} \xi_{k} e^{i\left(u, x_{k}\right)}\right|^{2} d \mu(u) \geq 0, \quad \xi=\left\{\xi_{k}\right\}_{k=1}^{m} \subset \mathbb{C} .
$$

If $Q(\xi)=0$, then the function $g(u):=\sum_{k=1}^{m} \xi_{k} e^{i\left(u, x_{k}\right)}$ equals 0 on supp $\mu$ and therefore equals 0 on $S_{n}^{r}(0)$. Finally, Lemma 3.7 yields that $\xi_{k}=0, k \in\{1, . ., m\}$.

Definition 3.10 A function $f(\cdot) \in C[0, \infty) \cap C^{\infty}(0,+\infty)$ is called completely monotonic function on $[0, \infty)$ of the class $M[0, \infty)$ if the inequality $(-1)^{k} f^{(k)}(t) \geq 0$ holds for all $k \in \mathbb{Z}_{+}$and $t>0$.

Schoenberg noted in [30, 31] that the function $f(\cdot) \in \bigcap_{n \in \mathbb{N}} \Phi_{n}$ if and only if $f(\sqrt{\cdot}) \in M[0, \infty)$. Thus, it is easily implied by Schoenberg theorem that $f(\cdot) \in M[0, \infty)$ yields $f(\cdot) \in \bigcap_{n \in \mathbb{N}} \Phi_{n}$.

Corollary 3.11 33. Theorem 7.14] If $f(\cdot) \in M[0, \infty)$ and $f(\cdot) \not \equiv$ const on $[0,+\infty)$, then the function $f(|\cdot|)$ is strictly positive definite on $\mathbb{R}^{n}$ for any $n \in \mathbb{N}$.

Remark 3.12 (i) In [33, Lemma 6.7] assertion of Lemma 3.7 was proven provided that the equality 3.5] holds on a certain open subset of $\mathbb{R}$.

(ii) Theorem 3.6] was formulated in [33, Theorem 6.18] and [14, Theorem 3.7] under the additional condition $t^{n-1} f(t) \in L^{1}[0, \infty)$.

Example 3.13 Let us present some examples of strictly positive functions.

(1) Using the equality $\Gamma(2 p)=\frac{2^{2 p-1}}{\sqrt{\pi}} \Gamma(p) \Gamma(p+1 / 2)$, we obtain

$$
\Omega_{1}(t)=\cos t, \quad \Omega_{2}(t)=J_{0}(t), \quad \Omega_{3}(t)=\sin t / t .
$$

By Theorem 3.6, the functions $\Omega_{n}(s|x|)$ are strictly positive definite on $\mathbb{R}^{n}$ for any $s>0$ and $n \geq 2$.

(2) It is easily seen that the functions $e^{-t}$ and $\left(1-e^{-t}\right) / t=\int_{0}^{1} e^{-t s} d s$ are completely monotonic on $[0,+\infty)$. Thus, by Corollary 3.11 , the functions $e^{-|x|}$ and $\left(1-e^{-|x|}\right) /|x|$ are strictly positive definite on $\mathbb{R}^{n}$ for all $n \in \mathbb{N}$.

\section{Three-dimensional Schrödinger operator with point interactions}

\subsection{Boundary triplet and Weyl function}

First we define a boundary triplet for the operator $H^{*}$. Denote $r_{j}:=\left|x-x_{j}\right|, x \in \mathbb{R}^{3}$, let also $\sqrt{\cdot}$ stands for the branch of the corresponding multifunction defined on $\mathbb{C} \backslash \mathbb{R}_{+}$by the condition $\sqrt{1}=1$. 
Proposition 4.1 Let $H$ be the minimal Schrödinger operator defined by (1.2) and let $\xi_{0}:=\left\{\xi_{0 j}\right\}_{j=1}^{m}, \quad \xi_{1}:=$ $\left\{\xi_{1 j}\right\}_{j=1}^{m} \in \mathbb{C}^{m}$. Then the following assertions hold

(i) The operator $H$ is closed and symmetric. The deficiency indices of $H$ are $n_{ \pm}(H)=m$. The defect subspace $\mathfrak{N}_{z}:=\mathfrak{N}_{z}(H)$ is

$$
\mathfrak{N}_{z}=\left\{\sum_{j=1}^{m} c_{j} \frac{e^{i \sqrt{z} r_{j}}}{4 \pi r_{j}}: c_{j} \in \mathbb{C}, j \in\{1, \ldots, m\}\right\}, \quad z \in \mathbb{C} \backslash[0, \infty) .
$$

(ii) The adjoint operator $H^{*}$ is given by

$$
\begin{aligned}
& \operatorname{dom}\left(H^{*}\right)=\left\{f=\sum_{j=1}^{m}\left(\xi_{0 j} \frac{e^{-r_{j}}}{r_{j}}+\xi_{1 j} e^{-r_{j}}\right)+f_{H}: \xi_{0}, \xi_{1} \in \mathbb{C}^{m}, f_{H} \in \operatorname{dom}(H)\right\}, \\
& H^{*} f=-\sum_{j=1}^{m}\left(\xi_{0 j} \frac{e^{-r_{j}}}{r_{j}}+\xi_{1 j}\left(e^{-r_{j}}-\frac{2 e^{-r_{j}}}{r_{j}}\right)\right)-\Delta f_{H} .
\end{aligned}
$$

(iii) A totality $\Pi=\left\{\mathcal{H}, \Gamma_{0}, \Gamma_{1}\right\}$, where

$$
\begin{aligned}
& \mathcal{H}=\mathbb{C}^{m}, \quad \Gamma_{0} f:=\left\{\Gamma_{0 j} f\right\}_{j=1}^{m}=4 \pi\left\{\lim _{x \rightarrow x_{j}} f(x)\left|x-x_{j}\right|\right\}_{j=1}^{m}=4 \pi\left\{\xi_{0 j}\right\}_{j=1}^{m}, \\
& \Gamma_{1} f:=\left\{\Gamma_{1 j} f\right\}_{j=1}^{m}=\left\{\lim _{x \rightarrow x_{j}}\left(f(x)-\frac{\xi_{0 j}}{\left|x-x_{j}\right|}\right)\right\}_{j=1}^{m},
\end{aligned}
$$

forms a boundary triplet for $H^{*}$.

(iv) The operator $H_{0}=H^{*} \uparrow \operatorname{ker}\left(\Gamma_{0}\right)\left(=H_{0}^{*}\right)$ coincides with the free Hamiltonian,

$$
H_{0}=-\Delta, \quad \operatorname{dom}\left(H_{0}\right)=\operatorname{dom}(-\Delta)=W^{2,2}\left(\mathbb{R}^{3}\right) .
$$

Proof. (i) The statement $(i)$ of Proposition4.1] is well-known. It was obtained in the classical book [3](see Theorem 1.1.2).

(ii) Clearly, $e^{-r_{j}} \in W^{2,2}\left(\mathbb{R}^{3}\right) \subset \operatorname{dom}\left(H^{*}\right)$ for $j \in\{1, . ., m\}$.

Since the function $e^{-|x|}$ is strictly positive definite on $\mathbb{R}^{3}$ (Example 3.13 , the matrix $\left(e^{-\left|x_{k}-x_{j}\right|}\right)_{k, j=1}^{m}$ is positive definite. Therefore the functions $e^{-r_{j}}, j \in\{1, . ., m\}$ are linearly independent. Consider the operator $\widetilde{H}$ defined by

$$
\widetilde{H}:=H^{*}\left\lceil\operatorname{dom}(\widetilde{H}), \quad \operatorname{dom}(\widetilde{H})=\operatorname{dom}(H) \dot{+} \operatorname{span}\left\{e^{-r_{j}}\right\}_{j=1}^{m}=W^{2,2}\left(\mathbb{R}^{3}\right) .\right.
$$

Since $\operatorname{dom}(\widetilde{H})=\operatorname{dom}(-\Delta)=W^{2,2}\left(\mathbb{R}^{3}\right)$ and both operators $\widetilde{H}$ and $-\Delta$ are proper extensions of $H$, we have $\widetilde{H}=-\Delta$ and the operator $\widetilde{H}$ is self-adjoint.

Further, since the functions $\frac{e^{-r_{j}}}{4 \pi r_{j}} \in \mathfrak{N}_{-1}, j \in\{1, . ., m\}$ are linearly independent, and, by the second J. von Neumann formula,

$$
\operatorname{dim}\left(\operatorname{dom}\left(H^{*}\right) / \operatorname{dom}(\widetilde{H})\right)=n_{ \pm}(H)=m,
$$

representation (4.2) is proved.

(iii) Let $f, g \in \operatorname{dom}\left(H^{*}\right)$. By 44.2], we have

$$
f=\sum_{k=1}^{m} f_{k}+f_{H}, \quad f_{k}=\xi_{0 k} \frac{e^{-r_{k}}}{r_{k}}+\xi_{1 k} e^{-r_{k}}, \quad g=\sum_{k=1}^{m} g_{k}+g_{H}, \quad g_{k}=\eta_{0 k} \frac{e^{-r_{k}}}{r_{k}}+\eta_{1 k} e^{-r_{k}},
$$

where $f_{H}, g_{H} \in \operatorname{dom}(H)$, and $\xi_{0 k}, \xi_{1 k}, \eta_{0 k}, \eta_{1 k} \in \mathbb{C}, k \in\{1, . ., m\}$. 
Applying (4.4)-(4.5) to $f, g \in \operatorname{dom}\left(H^{*}\right)$, we obtain

$$
\begin{aligned}
& \Gamma_{0} f=4 \pi\left\{\xi_{0 j}\right\}_{j=1}^{m}, \quad \Gamma_{1} f=\left\{-\xi_{0 j}+\sum_{k \neq j} \xi_{0 k} \frac{e^{-\left|x_{j}-x_{k}\right|}}{\left|x_{j}-x_{k}\right|}+\sum_{k=1}^{m} \xi_{1 k} e^{-\left|x_{j}-x_{k}\right|}\right\}_{j=1}^{m}, \\
& \Gamma_{0} g=4 \pi\left\{\eta_{0 j}\right\}_{j=1}^{m}, \quad \Gamma_{1} g=\left\{-\eta_{0 j}+\sum_{k \neq j} \eta_{0 k} \frac{e^{-\left|x_{j}-x_{k}\right|}}{\left|x_{j}-x_{k}\right|}+\sum_{k=1}^{m} \eta_{1 k} e^{-\left|x_{j}-x_{k}\right|}\right\}_{j=1}^{m} .
\end{aligned}
$$

It is easily seen that

$$
\begin{aligned}
&\left(H^{*} f, g\right)-\left(f, H^{*} g\right)=\sum_{k, j=1}^{m}\left(\left(\xi_{0 j} H^{*}\left(\frac{e^{-r_{j}}}{r_{j}}\right), \eta_{1 k} e^{-r_{k}}\right)-\left(\xi_{0 j} \frac{e^{-r_{j}}}{r_{j}}, \eta_{1 k} H^{*}\left(e^{-r_{k}}\right)\right)\right. \\
&\left.+\left(\xi_{1 j} H^{*}\left(e^{-r_{j}}\right), \eta_{0 k} \frac{e^{-r_{k}}}{r_{k}}\right)-\left(\xi_{1 j} e^{-r_{j}}, \eta_{0 k} H^{*}\left(\frac{e^{-r_{k}}}{r_{k}}\right)\right)\right) .
\end{aligned}
$$

Using the second Green formula, we get

$$
\begin{aligned}
& \left(H^{*}\left(\frac{e^{-r_{j}}}{r_{j}}\right), e^{-r_{k}}\right)-\left(\frac{e^{-r_{j}}}{r_{j}}, H^{*}\left(e^{-r_{k}}\right)\right) \\
& =\lim _{r \rightarrow \infty}\left(\int_{B_{r}\left(x_{j}\right) \backslash B_{\frac{1}{r}}\left(x_{j}\right)}-\Delta\left(\frac{e^{-r_{j}}}{r_{j}}\right) e^{-r_{k}} \mathrm{dx}+\int_{B_{r}\left(x_{j}\right) \backslash B_{\frac{1}{r}}\left(x_{j}\right)} \frac{e^{-r_{j}}}{r_{j}} \Delta\left(e^{-r_{k}}\right) \mathrm{dx}\right) \\
& =\lim _{r \rightarrow \infty} \int_{S_{r}\left(x_{j}\right)}\left(-\frac{\partial}{\partial n}\left(\frac{e^{-r_{j}}}{r_{j}}\right) e^{-r_{k}}+\frac{e^{-r_{j}}}{r_{j}} \frac{\partial\left(e^{-r_{k}}\right)}{\partial n}\right) \mathrm{ds} \\
& \quad+\lim _{r \rightarrow \infty} \int_{S_{\frac{1}{r}}\left(x_{j}\right)}\left(\frac{\partial}{\partial n}\left(\frac{e^{-r_{j}}}{r_{j}}\right) e^{-r_{k}}-\frac{e^{-r_{j}}}{r_{j}} \frac{\partial\left(e^{-r_{k}}\right)}{\partial n}\right) \mathrm{ds}=-4 \pi e^{-\left|x_{j}-x_{k}\right|,},
\end{aligned}
$$

where $n$ stands for the exterior normal vector to $S_{r}\left(x_{j}\right)$ and $S_{\frac{1}{r}}\left(x_{j}\right)$, respectively.

Indeed, noticing that $\frac{\partial}{\partial n}\left(\frac{e^{-r_{j}}}{r_{j}}\right)=-\frac{e^{-r_{j}}}{r_{j}}\left(1+\frac{1}{r_{j}}\right)$, we can easily show that the first integral in the formula (4.8) tends to zero as $r$ tends to infinity. Further,

$$
\begin{aligned}
& \lim _{r \rightarrow \infty} \int_{S_{\frac{1}{r}}\left(x_{j}\right)} \frac{\partial}{\partial n}\left(\frac{e^{-r_{j}}}{r_{j}}\right) e^{-r_{k}} \mathrm{ds}=\lim _{r \rightarrow \infty} \int_{S_{\frac{1}{r}}(0)} \frac{\partial}{\partial n}\left(\frac{e^{-|y|}}{|y|}\right) e^{-\left|y+x_{j}-x_{k}\right|} \mathrm{ds} \\
& \quad=-\lim _{r \rightarrow \infty}\left[\frac{4 \pi}{r^{2}} e^{-1 / r} r(1+r) e^{-\left|y^{*}+x_{j}-x_{k}\right|}\right]=-4 \pi \lim _{y^{*} \rightarrow 0} e^{-\left|y^{*}+x_{j}-x_{k}\right|}=-4 \pi e^{-\left|x_{j}-x_{k}\right|},
\end{aligned}
$$

and

$$
\begin{aligned}
& \lim _{r \rightarrow \infty} \int_{S_{\frac{1}{r}}\left(x_{j}\right)} \frac{e^{-r_{j}}}{r_{j}} \frac{\partial\left(e^{-r_{k}}\right)}{\partial n} \mathrm{ds}=\lim _{r \rightarrow \infty} \int_{S_{\frac{1}{r}}(0)} \frac{e^{-|y|}}{|y|} \frac{\partial\left(e^{-\left|y+x_{j}-x_{k}\right|}\right)}{\partial n} \mathrm{ds} \\
& \quad=\lim _{r \rightarrow \infty}\left[\left.\frac{4 \pi}{r^{2}} e^{-1 / r} r \frac{\partial\left(e^{-\left|y+x_{j}-x_{k}\right|}\right)}{\partial n}\right|_{y=y^{* *}}\right]=0, \quad y^{*} \in S_{\frac{1}{r}}(0), y^{* *} \in S_{\frac{1}{r}}(0) .
\end{aligned}
$$


Finally, by (4.7),

$$
\begin{aligned}
\left(H^{*} f, g\right)-\left(f, H^{*} g\right)=4 \pi \sum_{k, j=1}^{m} & \left(-\xi_{0 j} \bar{\eta}_{1 k} e^{-\left|x_{j}-x_{k}\right|}+\xi_{1 j} \bar{\eta}_{0 k} e^{-\left|x_{j}-x_{k}\right|}\right) \\
= & \sum_{j=1}^{m}\left(\Gamma_{1 j} f, \Gamma_{0 j} g\right)-\left(\Gamma_{0 j} f, \Gamma_{1 j} g\right)=\left(\Gamma_{1} f, \Gamma_{0} g\right)-\left(\Gamma_{0} f, \Gamma_{1} g\right) .
\end{aligned}
$$

Thus, the Green identity is satisfied. Let us show that that the mapping $\Gamma=\left(\Gamma_{0}, \Gamma_{1}\right)^{\top}$ is surjective. Let $\left(h_{0}, h_{1}\right)^{\top} \in \mathcal{H} \oplus \mathcal{H}$, where $h_{0}=\left\{h_{0 j}\right\}_{j=1}^{m}, h_{1}=\left\{h_{1 j}\right\}_{j=1}^{m}$ are vectors from $\mathbb{C}^{m}$. According to [4.2), any $f \in \operatorname{dom}\left(H^{*}\right)$ admits the representation $f=f_{H}+\sum_{j=1}^{m}\left(\xi_{0 j} \frac{e^{-r_{j}}}{r_{j}}+\xi_{1 j} e^{-r_{j}}\right)$. Let us put

$$
E_{0}:=\left(\frac{e^{-\left|x_{k}-x_{j}\right|}}{\left|x_{k}-x_{j}\right|-\delta_{k j}}\right)_{j, k=1}^{m}, \quad E_{1}:=\left(e^{-\left|x_{k}-x_{j}\right|}\right)_{k, j=1}^{m},
$$

where $\delta_{k j}$ denotes the Kronecker symbol. Invertibility of the matrix $E_{1}$ has been already established above. Therefore setting $\xi_{0}=\frac{1}{4 \pi} h_{0}$ and $\xi_{1}=E_{1}^{-1}\left(h_{1}-\frac{1}{4 \pi} E_{0} h_{0}\right)$, we get $\Gamma_{0} f=h_{0}$ and $\Gamma_{1} f=h_{1}$. Thus, $\Pi$ is a boundary triplet for $H^{*}$.

(iv) This statement easily follows from (4.2), (4.4) and (4.6).

Combining Proposition 2.2 with formulas [4.2], 4.7), we arrive at the following proposition.

Proposition 4.2 Let $H$ be the minimal Schrödinger operator defined by (1.2), $\Pi=\left\{\mathcal{H}, \Gamma_{0}, \Gamma_{1}\right\}$ a boundary triplet for $H^{*}$ defined by (4.5) and let the matrices $E_{0}, E_{1}$ be defined by [4.9]. Then the set of self-adjoint extensions $\widetilde{H} \in \mathrm{Ext}_{H}$ is parameterized as

$$
\widetilde{H}=H_{\Theta}=H^{*}\left\lceil\left\{f=\sum_{j=1}^{m}\left(\xi_{0 j} \frac{e^{-r_{j}}}{r_{j}}+\xi_{1 j} e^{-r_{j}}\right)+f_{H} \in \operatorname{dom}\left(H^{*}\right):\left\{\Gamma_{0} f, \Gamma_{1} f\right\} \in \Theta\right\},\right.
$$

where $\Theta$ runs through the set of all self-adjoint linear relations in $\mathcal{H}$. Moreover, $\Theta_{\infty}$ and $\Theta_{\mathrm{op}}$ are defined by

$$
\begin{aligned}
& \Theta_{\infty}=\left\{0, \mathcal{H}_{\infty}\right\}=\left\{\left\{0, E_{1} \xi_{1}^{\prime \prime}\right\}: \xi_{1}^{\prime \prime} \perp E_{1} \xi_{0}, \xi_{0} \in \mathcal{H}_{\mathrm{op}}\right\}, \\
& \Theta_{\mathrm{op}}=\left\{\left\{4 \pi \xi_{0}, E_{0} \xi_{0}+E_{1} \xi_{1}^{\prime}\right\}: \xi_{0} \in \mathcal{H}_{\mathrm{op}}, \xi_{1}^{\prime}=E_{1}^{-1}\left(4 \pi B \xi_{0}-E_{0} \xi_{0}\right)\right\},
\end{aligned}
$$

where $B=B^{*} \in\left[\mathcal{H}_{\mathrm{op}}\right]$. In particular, $\widetilde{H}=H_{\Theta}$ is disjoint with $H_{0}$ if and only if $\operatorname{dom}(\Theta)=\mathbb{C}^{m}$. In this case $\Theta=\Theta_{\mathrm{op}}=g r B$.

Proof. By (2.2), any self-adjoint extension $H_{\Theta}$ is parameterized as follows

$$
\begin{aligned}
& H_{\Theta}=H^{*}\left\lceil\operatorname{dom}\left(H_{\Theta}\right),\right. \\
& \operatorname{dom}\left(H_{\Theta}\right)=\left\{f=\sum_{j=1}^{m}\left(\xi_{0 j} \frac{e^{-r_{j}}}{r_{j}}+\xi_{1 j} e^{-r_{j}}\right)+f_{H}:\left\{4 \pi \xi_{0}, E_{0} \xi_{0}+E_{1} \xi_{1}\right\} \in \Theta=\Theta^{*}\right\} .
\end{aligned}
$$

It is easily seen that self-adjointness of the relation $\Theta$ is equivalent to the condition $\left(E_{1} \xi_{1}, \xi_{0}\right)=\left(\xi_{0}, E_{1} \xi_{1}\right)$. Since $\mathcal{H}_{\infty}=\operatorname{mul}(\Theta) \perp \operatorname{dom}(\Theta)=\mathcal{H}_{\mathrm{op}}$, this condition is equivalent to 4.11- 44.12). For instance, it follows from (4.11) and (4.12) that $\left(E_{1} \xi_{1}^{\prime \prime}, \xi_{0}\right)=0=\left(\xi_{0}, E_{1} \xi_{1}^{\prime \prime}\right)$ and

$$
\left(E_{1} \xi_{1}^{\prime}, \xi_{0}\right)=\left(4 \pi B \xi_{0}-E_{0} \xi_{0}, \xi_{0}\right)=\left(\xi_{0}, 4 \pi B \xi_{0}-E_{0} \xi_{0}\right)=\left(\xi_{0}, E_{1} \xi_{1}^{\prime}\right)
$$

Hence $\left(E_{1} \xi_{1}, \xi_{0}\right)=\left(\xi_{0}, E_{1} \xi_{1}\right)$. The arguments can be reversed. 
Remark 4.3 Note that the $m$-parametric family $H_{X, \alpha}^{(3)}$ investigated in [3, Theorem 1.1.1] is parameterized by the set of diagonal matrices $B_{\alpha}:=\operatorname{diag}\left(\alpha_{1}, . ., \alpha_{m}\right)=B_{\alpha}^{*}$. Namely, in this case, we put in 4.13$) \Theta=\Theta_{\mathrm{op}}=$ $B_{\alpha}$ and

$$
H_{X, \alpha}^{(3)}=H^{*} \uparrow\left\{f=\sum_{j=1}^{m} \xi_{0 j} \frac{e^{-r_{j}}}{r_{j}}+\sum_{k, j=1}^{m} b_{j k}(\alpha) \xi_{0 k} e^{-r_{j}}+f_{H}\right\}
$$

where $\widetilde{B}_{\alpha}=\left(b_{j k}(\alpha)\right)_{j, k=1}^{m}=E_{1}^{-1}\left(4 \pi B_{\alpha}-E_{0}\right)$.

Proposition 4.4 Let $H$ be the minimal Schrödinger operator defined by 1.2 and let $\Pi=\left\{\mathcal{H}, \Gamma_{0}, \Gamma_{1}\right\}$ be the boundary triplet for $H^{*}$ defined by (4.4)-(4.5). Then

(i) the corresponding Weyl function $M(\cdot)$ is

$$
M(z)=\left(\frac{i \sqrt{z}}{4 \pi} \delta_{j k}+\widetilde{G}_{\sqrt{z}}\left(x_{j}-x_{k}\right)\right)_{j, k=1}^{m}, \quad z \in \mathbb{C}_{+},
$$

where $\widetilde{G}_{\sqrt{z}}(x)=\left\{\begin{array}{ll}\frac{e^{i \sqrt{z}|x|}}{4 \pi|x|}, & x \neq 0 ; \\ 0, & x=0 .\end{array}\right.$ and $\delta_{k j}$ denotes the Kronecker symbol;

(ii) the corresponding $\gamma(\cdot)$-field is given by

$$
\gamma(z)\left\{a_{j}\right\}_{j=1}^{m}=\sum_{j=1}^{m} a_{j} \frac{e^{i \sqrt{z} r_{j}}}{4 \pi r_{j}}
$$

Proof. Let $f_{z} \in \mathfrak{N}_{z}$. By (4.1), $f_{z}=\sum_{j=1}^{m} a_{j} \frac{e^{i \sqrt{z} r_{j}}}{4 \pi r_{j}}, a_{j} \in \mathbb{C}$. Applying $\Gamma_{0}$ and $\Gamma_{1}$ to $f_{z}$, we get

$$
\Gamma_{0} f_{z}=\left\{a_{j}\right\}_{j=1}^{m}, \quad \Gamma_{1} f_{z}=\left\{a_{j} \frac{i \sqrt{z}}{4 \pi}+\sum_{k \neq j} a_{k} \frac{e^{i \sqrt{z}\left|x_{j}-x_{k}\right|}}{4 \pi\left|x_{j}-x_{k}\right|}\right\}_{j=1}^{m}, \quad z \in \mathbb{C} \backslash[0, \infty) .
$$

Substituting these formulas in (2.3) (cf. Definition 2.6), we arrive at 4.15).

The second statement follows immediately from (4.16) and 2.3.

Remark $4.5(i)$ In the case $m=1$, description 44.1] of $\mathfrak{N}_{z}$ was obtained by Lyantse and Majorga [22], Lemma 4.1].

(ii) The first construction of the boundary triplet, in the case $m=1$, goes apparently back to the paper by Lyantse and Majorga [22, Theorem 2.1]. Slightly different boundary triplet was obtained in [10, Section 5.4]. Another construction of the boundary triplet in the situation of general elliptic operator with boundary conditions on a set of zero Lebesgue measure was obtained by A. Kochubei [21]. However this construction is not suitable for our purpose.

(iii) The Weyl function in the form (4.15) has appeared in Krein's formula for resolvent of $H_{X, \alpha}^{(3)}$ (see [3, chapter II.1]). In this connection we also mention the paper by Posilicano [28, Example 5.3]. Note also that, in the case $m=1$, the Weyl function was computed in [10, Section 5.4] and without boundary triplets in [7, Section 10.3].

\subsection{Spectrum of the self-adjoint extensions of the minimal Schrödinger operator $H$}

In what follows we need the following lemma.

Lemma 4.6 Let $H^{*}$ be defined by (4.2)-(4.3). Then $0 \notin \sigma_{p}\left(H^{*}\right)$.

Proof. Let $f_{0} \in \operatorname{dom}\left(H^{*}\right)$ and $H^{*} f_{0}=0$. Observing that $\frac{e^{-r_{j}}-1}{r_{j}} \in W_{\text {loc }}^{2,2}\left(\mathbb{R}^{3}\right)$ for any $j \in\{1, . ., m\}$, we obtain from (4.2) that $f_{0}$ admits a representation

$$
f_{0}=\sum_{j=1}^{m} \xi_{0 j} \frac{1}{r_{j}}+g, \quad \text { with } \quad g:=\sum_{j=1}^{m}\left(\xi_{0 j} \frac{e^{-r_{j}}-1}{r_{j}}+\xi_{1 j} e^{-r_{j}}\right)+f_{H} \in W_{\mathrm{loc}}^{2,2}\left(\mathbb{R}^{3}\right) .
$$


By definition of $H^{*}$, we get $\left(f_{0}, \Delta \varphi\right)=0, \varphi \in C_{0}^{\infty}\left(\mathbb{R}^{3} \backslash X\right)$, i.e., $f_{0}$ is a week solution of the equation $\Delta f_{0}=0$. By regularity theorem (Weyl's lemma [17, chapter 8]), $f_{0}(\cdot)$ is harmonic function in $\mathbb{R}^{3} \backslash X$. Since $\left(\Delta \frac{1}{r_{j}}\right)(x)=0$ and $\left(H^{*} f_{0}\right)(x)=-\left(\Delta f_{0}\right)(x)=0$ for $x \notin X$, we get that $g(x)$ is harmonic function for $x \notin X$ and continuous on $\mathbb{R}^{3}$ by Sobolev embedding theorem.

It follows from definition 4.17) that $g(\cdot)$ is bounded. Therefore, by desingularization theorem (see [24, chapter $\mathrm{IV}, \S 3])$, it can be extended by continuity to $X$, and the extended function is harmonic on $\mathbb{R}^{3}$. Therefore, by the Liouville theorem for harmonic functions, $g(x)=$ const, $x \in \mathbb{R}^{3}$. Since $g(\cdot) \in L^{2}\left(\mathbb{R}^{3}\right)$, we have $g(\cdot) \equiv 0$ in $\mathbb{R}^{3}$. Observing that $1 / r_{j} \notin L^{2}\left(\mathbb{R}^{3}\right), j \in\{1, . ., m\}$ and that the functions $1 / r_{j}$ are linearly independent, we have $f_{0}(\cdot) \equiv 0$ in $\mathbb{R}^{3}$.

In the following theorem we describe spectrum of the self-adjoint extensions of $H$.

Theorem 4.7 Let $H$ be the minimal Schrödinger operator defined by (1.2) and $\Pi$ be the boundary triplet for $H^{*}$ defined by (4.4)-(4.5). Let also $M(\cdot)$ be the corresponding Weyl function defined by (4.15). Assume that $H_{\Theta}=H_{\Theta}^{*} \in$ Ext $_{H}$ defined by (4.10). Then the following assertions hold.

(i) The extension $H_{\Theta}$ has purely absolutely continuous non-negative spectrum of infinite multiplicity.

(ii) Point spectrum of the extension $H_{\Theta}$ consists of at most $m$ negative eigenvalues (counting multiplicities), $\kappa_{-}\left(H_{\Theta}\right) \leq m$. Moreover, $z \in \sigma_{p}\left(H_{\Theta}\right) \cap \mathbb{R}_{-}$if and only if $0 \in \sigma_{p}(\Theta-M(z))$, i.e.,

$$
z \in \sigma_{p}\left(H_{\Theta}\right) \cap \mathbb{R}_{-} \Longleftrightarrow 0 \in \sigma_{p}\left(\Theta-\left(\frac{i \sqrt{z}}{4 \pi} \delta_{j k}+\widetilde{G}_{\sqrt{z}}\left(x_{j}-x_{k}\right)\right)_{j, k=1}^{m}\right) .
$$

The corresponding eigenfunctions $\psi_{z}$ have the form

$$
\psi_{z}=\sum_{j=1}^{m} a_{j} \frac{e^{i \sqrt{z} r_{j}}}{4 \pi r_{j}}, \quad \text { where } \quad\left(a_{1}, . ., a_{m}\right)^{\top} \in \operatorname{ker}(\Theta-M(z)) .
$$

(iii) The number of negative eigenvalues of the self-adjoint extension $H_{\Theta}$ equals the number of negative eigenvalues of the relation $\Theta-M(0), \kappa_{-}\left(H_{\Theta}\right)=\kappa_{-}(\Theta-M(0))$, i.e.,

$$
\kappa_{-}\left(H_{\Theta}\right)=\kappa_{-}\left(\Theta-\left(\frac{1-\delta_{j k}}{4 \pi\left|x_{k}-x_{j}\right|+\delta_{j k}}\right)_{j, k=1}^{m}\right) .
$$

Proof. $(i)$ Note that symmetric operator $H$ is not simple since the multiplicity of the spectrum of its extension $H_{0}=H_{0}^{*}$ is infinite. Therefore $H$ admits the representation $H=\widehat{H} \oplus H_{s}$, where $\widehat{H}$ and $H_{s}$ are the simple and the self-adjoint part of $H$, respectively, defined by

$$
\begin{aligned}
& \widehat{H}=H \uparrow P_{\widehat{\mathfrak{H}}}(\operatorname{dom}(H)), \quad \widehat{\mathfrak{H}}=\overline{\operatorname{span}}\left\{\mathfrak{N}_{z}: z \in \mathbb{C} \backslash \mathbb{R}\right\}, \\
& H_{s}=H \uparrow P_{\mathfrak{H}_{s}}(\operatorname{dom}(H))=H_{s}^{*}, \quad \mathfrak{H}_{s}=L^{2}\left(\mathbb{R}^{3}\right) \ominus \widehat{\mathfrak{H}},
\end{aligned}
$$

where $P_{\widehat{\mathfrak{H}}}$ and $P_{\mathfrak{H}_{s}}$ are orthogonal projectors onto $\widehat{\mathfrak{H}}$ and $\mathfrak{H}_{s}$, respectively. Clearly, a totality $\widehat{\Pi}=\left\{\mathcal{H}, \widehat{\Gamma}_{0}, \widehat{\Gamma}_{1}\right\}=$ : $\left\{\mathcal{H}, \Gamma_{0} \uparrow \widehat{\mathfrak{H}}, \Gamma_{1} \uparrow \widehat{\mathfrak{H}}\right\}$ forms a boundary triplet for $\widehat{H}^{*}$. Then the operator $H_{0}$ takes the form $H_{0}=\widehat{H}_{0} \oplus H_{s}$, where $\widehat{H}_{0}=\widehat{H}^{*} \uparrow \operatorname{ker}\left(\widehat{\Gamma}_{0}\right)=\widehat{H}_{0}^{*}$.

For simplicity, we confine ourselves to the case of realizations $H_{\Theta}$ disjoint with $H_{0}$, i.e., $\operatorname{dom}\left(H_{\Theta}\right) \cap \operatorname{dom}\left(H_{0}\right)=$ $\operatorname{dom}(H)$. Then $H_{\Theta}=H_{B}=H^{*} \uparrow \operatorname{ker}\left(\Gamma_{1}-B \Gamma_{0}\right)$ with $B=B^{*} \in[\mathcal{H}]$. Thereby $H_{B}=\widehat{H}_{B} \oplus H_{s}$, $\widehat{H}_{B}=\widehat{H}^{*} \uparrow \operatorname{ker}\left(\widehat{\Gamma}_{0}^{B}\right)$, where corresponding boundary triplet $\widehat{\Pi}_{B}=\left\{\mathcal{H}^{B}, \widehat{\Gamma}_{0}^{B}, \widehat{\Gamma}_{1}^{B}\right\}$ is defined by

$$
\mathcal{H}^{B}=\mathcal{H}, \quad \widehat{\Gamma}_{0}^{B}=B \widehat{\Gamma}_{0}-\widehat{\Gamma}_{1}, \quad \widehat{\Gamma}_{1}^{B}=\widehat{\Gamma}_{0} .
$$

The appropriate Weyl function is $M_{B}(z)=(B-M(z))^{-1}$. Moreover, it is easily seen that

$$
\operatorname{Im}\left(M_{B}(z)\right)=(B-M(z))^{-1} \operatorname{Im}(M(z))\left(B-M^{*}(z)\right)^{-1}, \quad z \in \mathbb{C} \backslash \sigma_{p}\left(H_{B}\right) .
$$


It follows from (4.15) that the strong limit $M(x+i 0)=s-\lim _{y \downarrow 0} M(x+i y)$ exists for all $x \in \mathbb{R}$ and

$$
M(x+i 0)=\left(\frac{i \sqrt{x}}{4 \pi} \delta_{k j}+\frac{e^{i \sqrt{x}\left|x_{k}-x_{j}\right|}-\delta_{k j}}{4 \pi\left|x_{k}-x_{j}\right|+\delta_{k j}}\right)_{j, k=1}^{m}, \quad x \in \mathbb{R} .
$$

Therefore

$$
\operatorname{Im}(M(x+i 0))=\left(\frac{\sqrt{x}}{4 \pi} \delta_{k j}+\frac{\sin \left(\sqrt{x}\left|x_{k}-x_{j}\right|\right)}{4 \pi\left|x_{k}-x_{j}\right|+\delta_{k j}}\right)_{j, k=1}^{m}, \quad x \in \mathbb{R}_{+}
$$

and $\operatorname{Im}(M(x+i 0))=0$ for $x \leq 0$. Combining this fact with 4.20 , we conclude that

$$
\operatorname{Im}\left(M_{B}(x+i 0)\right)=(B-M(x+i 0))^{-1} \operatorname{Im}(M(x+i 0))\left(B-M^{*}(x+i 0)\right)^{-1}, \quad x \in \mathbb{R} \backslash \sigma_{p}\left(H_{B}\right) .
$$

Since the functions $\frac{\sin s x}{s x} \in \Phi_{3}, s>0$ (see Example 3.13), we conclude that the matrix $\operatorname{Im}(M(x+i 0)) / \sqrt{x}$ is positive definite for all $x>0$. Hence the matrix $\operatorname{Im}\left(M_{B}(x+i 0)\right) / \sqrt{x}$ is also positive definite for every $x>0$. Thereby, the matrix $B-M(x+i 0)$ is invertible for all $x \in \mathbb{R}_{+}$and consequently $\sigma_{p}\left(\widehat{H}_{B}\right) \cap \mathbb{R}_{+}=\emptyset$. It also follows from (4.22) that the multiplicity function $d_{M_{B}(x)}$ is maximal for all $x>0$, i.e., $d_{M(x)}=d_{M_{B}(x)}=m$.

Therefore, by Proposition 2.9 $(i i), \widehat{H}_{0}^{a c}$ and $\widehat{H}_{B}^{a c}$ are unitarily equivalent. By Proposition2.9 $(i),(i i), \sigma_{s c}\left(\widehat{H}_{0}\right)=$ $\sigma_{p}\left(\widehat{H}_{0}\right)=\emptyset$ and $\sigma_{a c}\left(\widehat{H}_{0}\right)=[0, \infty)$. Since $H_{s}=H_{s}^{a c}$ and $\sigma_{a c}\left(H_{s}\right)=[0, \infty)($ see [29, Chapter XIII]), $\sigma_{a c}\left(H_{B}\right)=[0, \infty)$ and $\sigma_{p}\left(H_{B}\right) \cap \mathbb{R}_{+}=\emptyset$. Further, Proposition 2.9 $(i)$ and the equality $H_{s}=H_{s}^{a c}$ together yield $\sigma_{s c}\left(H_{B}\right) \cap \mathbb{R}_{+}=\emptyset$. Absence of negative continuous spectrum of $H_{B}$ follows from the relations $\widehat{H} \geq 0$ and $n_{ \pm}(\widehat{H})=m$ (see [25, chapter 4,§14]). To complete the proof it remains to apply Lemma4.6.

(ii) According to the decomposition $H=\widehat{H} \oplus H_{s}$, we have $H_{\Theta}=\widehat{H}_{\Theta} \oplus H_{s}$. Since $\mathbb{R}_{-} \subset \rho\left(H_{0}\right)$ and $H_{s} \geq 0$, Proposition 2.7 applied to the simple part $\widehat{H}$ of $H$ yields the equivalences

$$
z \in \sigma_{p}\left(H_{\Theta}\right) \cap \mathbb{R}_{-} \Longleftrightarrow z \in \sigma_{p}\left(\widehat{H}_{\Theta}\right) \cap \mathbb{R}_{-} \Longleftrightarrow 0 \in \sigma_{p}(\Theta-M(z)) .
$$

Combining this formula with formula (4.15) for the Weyl function yields (4.18). Formula (4.19) follows from (4.16) and Proposition2.7(ii) applied to the simple part $\widehat{H}$ of $H$.

It remains to note that the inequality $\kappa_{-}\left(H_{\Theta}\right)=\kappa_{-}\left(\widehat{H}_{\Theta}\right) \leq m$ is immediate from the fact that $H \geq 0$ and $n_{ \pm}(H)=n_{ \pm}(\widehat{H})=m$ (see [25, chapter $\left.\left.4, \S 14\right]\right)$.

(iii) Combining Proposition 2.8 $($ ii) with 4.15, we arrive at (iii).

Remark 4.8 Note that the invertibility of the matrix $\operatorname{Im}(M(x+i 0))$ 4.21 for all but finite number of $x \in \mathbb{R}_{+}$ can directly be extracted without involving positive definite functions theory. Clearly, the function $V(x):=$ $\operatorname{det}(\operatorname{Im}(M(x))) / \sqrt{x}$ admits holomorphic continuation on $\mathbb{C}$. Since $\lim _{z \rightarrow \infty} V(z)=I_{m}$, the number of zeroes of $V(\cdot)$ on $\mathbb{R}_{+}$is finite because of its analiticity.

Remark 4.9 (i) The description of absolutely continuous and point spectrum in the particular case of the family $H_{X, \alpha}^{(3)}$ defined by (4.14) was obtained in [3, Theorem 1.1.4] by another method.

(ii) Complete description of the negative spectrum of the $m$-parametric family $H_{X, \alpha}^{(3)}$ was recently obtained by O. Ogurisu in [26, section 2] by another method.

\subsection{Non-negative self-adjoint extensions of the minimal Schrödinger operator $H$}

Here we propose slightly different boundary triplet for $H^{*}$ and compute the corresponding Weyl function. It turns out that the new Weyl function is more convenient for the description of non-negative self-adjoint extensions of the minimal operator $H$ than the one constructed in Proposition 4.4.

Proposition 4.10 Let $H$ be the minimal Schrödinger operator defined by (1.2), let $\Pi$ be the boundary triplet for $H^{*}$ defined by (4.4)-(4.5), and let $M(\cdot)$ be the corresponding Weyl function defined by (4.15). Then the set of 
all non-negative self-adjoint extensions $H_{\Theta} \in E t_{H}$ is parameterized by

$$
H_{\Theta}=H^{*} \uparrow\left\{f=\sum_{k, j=1}^{m} b_{j k}^{\prime} \xi_{1 k} \frac{e^{-r_{j}}}{r_{j}}+\sum_{j=1}^{m} \xi_{1 j} e^{-r_{j}}+f_{H}, f_{H} \in \operatorname{dom}(H)\right\},
$$

where $B^{\prime}=\left(b_{k j}^{\prime}\right)_{k, j=1}^{m}=\frac{1}{4 \pi} B E_{1}$, with $E_{1}=\left(e^{-\left|x_{k}-x_{j}\right|}\right)_{k, j=1}^{m}$ and $B$ runs over the set of all matrices satisfying the condition $0<B<4 \pi\left(\left(\frac{1-e^{-\left|x_{k}-x_{j}\right|}-\delta_{j k}}{\left|x_{k}-x_{j}\right|-\delta_{j k}}\right)_{k, j=1}^{m}\right)^{-1}$.

Pro of. Alongside the triplet $\Pi=\left\{\mathcal{H}, \Gamma_{0}, \Gamma_{1}\right\}$ consider the new boundary triplet $\widetilde{\Pi}=\left\{\widetilde{\mathcal{H}}, \widetilde{\Gamma}_{0}, \widetilde{\Gamma}_{1}\right\}$ (cf. [12]),

$$
\widetilde{\mathcal{H}}:=\mathcal{H}, \quad \widetilde{\Gamma}_{0}:=\Gamma_{0}, \quad \widetilde{\Gamma}_{1}:=\Gamma_{1}-\frac{1}{4 \pi} E_{0} \Gamma_{0},
$$

where $E_{0}$ is defined by 4.9). Then $\widetilde{M}(z)=M(z)-\frac{1}{4 \pi} E_{0}$. Using 4.15), we obtain

$$
\widetilde{M}(0)=\frac{1}{4 \pi}\left(\frac{1-e^{-\left|x_{k}-x_{j}\right|}-\delta_{j k}}{\left|x_{k}-x_{j}\right|-\delta_{j k}}\right)_{k, j=1}^{m}
$$

By Proposition 2.8 non-negative self-adjoint extensions $H_{\Theta}$ are described by the condition $\Theta-\widetilde{M}(0) \geq 0$. Since the function $f(t)=\frac{1-e^{-t}}{t} \in \bigcap_{n \in \mathbb{N}} \Phi_{n}$ (see Example 3.13), the matrix $\widetilde{M}(0)$ is positive definite. Therefore $\Theta$ is also positive definite and inverse matrix $\Theta^{-1}=B$ exists and $H_{\Theta}=H^{*} \uparrow \operatorname{ker}\left(B \widetilde{\Gamma}_{1}-\widetilde{\Gamma}_{0}\right)$. Thus, by (2.2) and (4.2), the desired parametrization holds.

Remark 4.11 It should be noted that the above description is close to the following obtained by Yu. Arlinskii and E. Tsekanovskii (see [5, Theorem 5.1]) in the framework of another approach. Namely, any non-negative self-adjoint extension $\widetilde{H}$ of the minimal operator $H$ admits the representation

$$
\begin{gathered}
\operatorname{dom}(\widetilde{H})=\left\{f_{H}+\sum_{j=1}^{m} \xi_{j} \sqrt{\frac{\pi}{2}} \frac{e^{-\frac{r_{j}}{\sqrt{2}}}}{r_{j}} \sin \left(\frac{r_{j}}{\sqrt{2}}\right)+\sum_{k, j=1}^{m} u_{k j} \xi_{k} \sqrt{\frac{\pi}{2}} \frac{e^{-\frac{r_{j}}{\sqrt{2}}}}{r_{j}} \cos \left(\frac{r_{j}}{\sqrt{2}}\right)\right\} \\
f_{H} \in \operatorname{dom}(H), \quad\left(\xi_{1}, . ., \xi_{m}\right) \in \mathbb{C}^{m}
\end{gathered}
$$

where $\mathcal{U}=\left(u_{k j}\right)_{k, j=1}^{m}$ runs over the set of matrices satisfying the condition $0 \leq \mathcal{U} \mathcal{G} \leq \mathcal{G} \mathcal{W}_{0}^{-1} \mathcal{G}$ with $\mathcal{W}_{0}$ and $\mathcal{G}$ defined by, respectively,

$$
2 \pi^{2}\left(\frac{\delta_{j k}}{\sqrt{2}}+\frac{1-\exp \left(\frac{-r_{k j}}{\sqrt{2}}\right) \cos \frac{r_{k j}}{\sqrt{2}}}{r_{k j}+\delta_{j k}}\right)_{k, j=1}^{m}, 2 \pi^{2}\left(\frac{\delta_{j k}}{\sqrt{2}}+\frac{\exp \left(\frac{-r_{k j}}{\sqrt{2}}\right) \sin \frac{r_{k j}}{\sqrt{2}}}{r_{k j}+\delta_{j k}}\right)_{k, j=1}^{m}
$$

where $r_{k j}=\left|x_{k}-x_{j}\right|$.

\section{Two-dimensional Schrödinger operator with point interactions}

\subsection{Boundary triplet and Weyl function}

Let $H_{0}^{(1)}(\cdot)$ denote the Hankel function of the first kind and zero-order. It is known that the function $H_{0}^{(1)}(z)$ has the following asymptotic expansion at 0 (see formulas $(9.01)$ in [27, chapter 2,] and (5.03), (5.07) in [27, chapter 7])

$$
H_{0}^{(1)}(z)=1+\frac{2 i}{\pi}\left(\ln \left(\frac{z}{2}\right)-\psi(1)\right)+o(z), \quad|z| \rightarrow 0, \quad \psi(1)=\frac{\Gamma^{\prime}(1)}{\Gamma(1)}
$$


Proposition 5.1 Let $H$ be the minimal Schrödinger operator defined by (1.2) and $\xi_{0}, \xi_{1}$ be defined as in the previous case. Then the following assertions hold.

(i) The operator $H$ is closed and symmetric. The deficiency indices of $H$ are $n_{ \pm}(H)=m$. The defect subspace $\mathfrak{N}_{z}:=\mathfrak{N}_{z}(H)$ is

$$
\mathfrak{N}_{z}=\left\{\sum_{j=1}^{m} c_{j} \frac{i}{4} H_{0}^{(1)}\left(\sqrt{z} r_{j}\right): c_{j} \in \mathbb{C}, j \in\{1, \ldots, m\}\right\}, \quad z \in \mathbb{C} \backslash[0, \infty) .
$$

(ii) The domain of $H^{*}$ is defined by

$$
\begin{aligned}
& \operatorname{dom}\left(H^{*}\right)=\left\{f=\sum_{j=1}^{m}\left(\xi_{0 j} e^{-r_{j}} \ln \left(r_{j}\right)+\xi_{1 j} e^{-r_{j}}\right)+f_{H}: \xi_{0}, \xi_{1} \in \mathbb{C}^{m}, \quad f_{H} \in \operatorname{dom}(H)\right\}, \\
& H^{*} f=-\sum_{j=1}^{m}\left(\xi_{0 j} \frac{e^{-r_{j}}}{r_{j}}\left(r_{j} \ln \left(r_{j}\right)-\ln \left(r_{j}\right)-2\right)+\xi_{1 j} \frac{e^{-r_{j}}}{r_{j}}\left(1-r_{j}\right)\right)-\Delta f_{H} .
\end{aligned}
$$

(iii) A totality $\Pi=\left\{\mathcal{H}, \Gamma_{0}, \Gamma_{1}\right\}$, where

$$
\begin{aligned}
& \mathcal{H}=\mathbb{C}^{m}, \quad \Gamma_{0} f:=\left\{\Gamma_{0 j} f\right\}_{j=1}^{m}=-2 \pi\left\{\lim _{x \rightarrow x_{j}} \frac{f(x)}{\ln \left|x-x_{j}\right|}\right\}_{j=1}^{m}=2 \pi\left\{\xi_{0 j}\right\}_{j=1}^{m}, \\
& \Gamma_{1} f:=\left\{\Gamma_{1 j} f\right\}_{j=1}^{m}=\left\{\lim _{x \rightarrow x_{j}}\left(f(x)-\ln \left|x-x_{j}\right| \xi_{0 j}\right)\right\}_{j=1}^{m}, \quad f \in \operatorname{dom}\left(H^{*}\right),
\end{aligned}
$$

forms a boundary triplet for $H^{*}$.

(iv) The operator $H_{0}=H^{*} \uparrow \operatorname{ker}\left(\Gamma_{0}\right)\left(=H_{0}^{*}\right)$ coincides with the free Hamiltonian,

$$
H_{0}=-\Delta, \quad \operatorname{dom}\left(H_{0}\right)=\operatorname{dom}(-\Delta)=W^{2,2}\left(\mathbb{R}^{2}\right) .
$$

Proof. (i) First two statements are known (see, for instance, [3, chapter II.4]). Formula (5.2) is also known. However we present the proof for the sake of completeness. The inclusion $f_{j}=H_{0}^{(1)}\left(\sqrt{z} r_{j}\right) \in \mathfrak{N}_{z}, z \in$ $\mathbb{C} \backslash[0, \infty)$ is amount to saying that

$$
\left(H_{0}^{(1)}\left(\sqrt{z} r_{j}\right),(H-\bar{z}) \varphi\right)=0, \quad \varphi \in \operatorname{dom}(H), \quad j \in\{1, . ., m\} .
$$

Since $\frac{i}{4} H_{0}^{(1)}\left(\sqrt{z}\left|x-x^{\prime}\right|\right)$ is the kernel of the free Hamiltonian resolvent $R_{z}\left(H_{0}\right)$ (see [3, chapter I.5]), we get

$$
\left(\frac{i}{4} H_{0}^{(1)}\left(\sqrt{z}\left|x-x^{\prime}\right|\right),\left(H_{0}-\bar{z}\right) \bar{\psi}\right)=R_{z}\left(H_{0}\right)\left(\left(H_{0}-z\right) \psi\right)=\psi\left(x^{\prime}\right), \quad \psi \in \operatorname{dom}\left(H_{0}\right) .
$$

Hence for $\varphi \in \operatorname{dom}(H)$

$$
\left(H_{0}^{(1)}\left(\sqrt{z} r_{j}\right),(H-\bar{z}) \varphi\right)=\left(H_{0}^{(1)}\left(\sqrt{z} r_{j}\right),\left(H_{0}-\bar{z}\right) \varphi\right)=-4 i \bar{\varphi}\left(x_{j}\right)=0,
$$

which proves 5.7). Thus, $f_{j} \in \operatorname{ran}(H-\bar{z})^{\perp}=\mathfrak{N}_{z}, j \in\{1, . ., m\}$.

Clearly, the functions $\left\{H_{0}^{(1)}\left(\sqrt{z} r_{j}\right)\right\}_{j=1}^{m}$, are linearly independent. Indeed, otherwise we have the equality

$$
\sum_{j=1}^{m} c_{j} H_{0}^{(1)}\left(\sqrt{z} r_{j}\right)=0, \quad \text { with } \quad c_{j} \in \mathbb{C}, \quad \sum_{j=1}^{m}\left|c_{j}\right| \neq 0 .
$$

Let for definiteness $c_{1} \neq 0$. Multiplying $(5.8)$ by $\frac{1}{\ln \left(r_{1}\right)}$, then passing to the limit as $x$ tends to $x_{1}$ and taking the asymptotic formula (5.1) into account, we get $c_{1}=0$. The contradiction proves (5.2).

(ii) It is known (see [3, 4]) that

$$
\operatorname{dom}\left(H^{*}\right)=\left\{f \in L^{2}\left(\mathbb{R}^{2}\right) \cap W_{\text {loc }}^{2,2}\left(\mathbb{R}^{2} \backslash X\right): \Delta f \in L^{2}\left(\mathbb{R}^{2}\right)\right\} .
$$


Obviously, the functions $f_{j}=e^{-r_{j}} \ln \left(r_{j}\right)$ and $g_{j}=e^{-r_{j}}, j \in\{1, . ., m\}$ belong to $\operatorname{dom}\left(H^{*}\right)$. Their linear independency might be derived as in 3D case. Since $\operatorname{dim}\left(\operatorname{dom}\left(H^{*}\right) / \operatorname{dom}(H)\right)=2 m$, the $\operatorname{domain} \operatorname{dom}\left(H^{*}\right)$ takes the form (5.3).

(iii) Let $f, g \in \operatorname{dom}\left(H^{*}\right)$. By assertion $(i)$, we have

$$
\begin{aligned}
& f=\sum_{k=1}^{m} f_{k}+f_{H}, \quad f_{k}=\xi_{0 k} e^{-r_{k}} \ln \left(r_{k}\right)+\xi_{1 k} e^{-r_{k}}, \quad \text { and } \\
& g=\sum_{k=1}^{m} g_{k}+g_{H}, \quad g_{k}=\eta_{0 k} e^{-r_{k}} \ln \left(r_{k}\right)+\eta_{1 k} e^{-r_{k}},
\end{aligned}
$$

where $f_{H}, g_{H} \in \operatorname{dom}(H)$, and $\xi_{0 k}, \xi_{1 k}, \eta_{0 k}, \eta_{1 k} \in \mathbb{C}, k \in\{1, . ., m\}$.

Applying $\Gamma_{0}, \Gamma_{1}$ to $f$ and $g$, we obtain

$$
\begin{aligned}
& \Gamma_{0} f=2 \pi\left\{\xi_{0 j}\right\}_{j=1}^{m}, \quad \Gamma_{1} f=\left\{\sum_{k \neq j} \xi_{0 k} e^{-\left|x_{j}-x_{k}\right|} \ln \left|x_{j}-x_{k}\right|+\sum_{k=1}^{m} \xi_{1 k} e^{-\left|x_{j}-x_{k}\right|}\right\}_{j=1}^{m}, \\
& \Gamma_{0} g=2 \pi\left\{\eta_{0 j}\right\}_{j=1}^{m}, \quad \Gamma_{1} g=\left\{\sum_{k \neq j} \eta_{0 k} e^{-\left|x_{j}-x_{k}\right|} \ln \left|x_{j}-x_{k}\right|+\sum_{k=1}^{m} \eta_{1 k} e^{-\left|x_{j}-x_{k}\right|}\right\}_{j=1}^{m} .
\end{aligned}
$$

Left-hand side of the Green identity 2.1 takes the form

$$
\begin{aligned}
\left(H^{*} f, g\right)-\left(f, H^{*} g\right)= & \sum_{k, j=1}^{m}\left(\left(\xi_{0 j} H^{*}\left(e^{-r_{j}} \ln \left(r_{j}\right)\right), \eta_{1 k} e^{-r_{k}}\right)-\left(\xi_{0 j} e^{-r_{j}} \ln \left(r_{j}\right), \eta_{1 k} H^{*}\left(e^{-r_{k}}\right)\right)\right. \\
& \left.+\left(\xi_{1 j} H^{*}\left(e^{-r_{j}}\right), \eta_{0 k} e^{-r_{k}} \ln \left(r_{k}\right)\right)-\left(\xi_{1 j} e^{-r_{j}}, \eta_{0 k} H^{*}\left(e^{-r_{k}} \ln \left(r_{k}\right)\right)\right)\right) .
\end{aligned}
$$

Applying the second Green formula yields

$$
\begin{aligned}
& \left(H^{*}\left(e^{-r_{j}} \ln \left(r_{j}\right)\right), e^{-r_{k}}\right)-\left(e^{-r_{j}} \ln \left(r_{j}\right), H^{*}\left(e^{-r_{k}}\right)\right)= \\
& \lim _{r \rightarrow \infty} \int_{B_{r}\left(x_{j}\right) \backslash B_{\frac{1}{r}}\left(x_{j}\right)}\left(-\Delta\left(e^{-r_{j}} \ln \left(r_{j}\right)\right) e^{-r_{k}}+e^{-r_{j}} \ln \left(r_{j}\right) \Delta\left(e^{-r_{k}}\right)\right) \mathrm{dx}=-2 \pi e^{-\left|x_{k}-x_{j}\right|} .
\end{aligned}
$$

By (5.9) and (5.10), we obtain

$$
\begin{aligned}
\left(H^{*} f, g\right)-\left(f, H^{*} g\right)=2 \pi \sum_{k, j=1}^{m} & \left(-\xi_{0 j} \bar{\eta}_{1 k} e^{-\left|x_{j}-x_{k}\right|}+\xi_{1 j} \bar{\eta}_{0 k} e^{-\left|x_{j}-x_{k}\right|}\right) \\
= & \sum_{j=1}^{m}\left(\Gamma_{1 j} f, \Gamma_{0 j} g\right)-\left(\Gamma_{0 j} f, \Gamma_{1 j} g\right)=\left(\Gamma_{1} f, \Gamma_{0} g\right)-\left(\Gamma_{0} f, \Gamma_{1} g\right)
\end{aligned}
$$

Thus, the Green identity is verified. From (5.3) it follows that the mapping $\Gamma=\left(\Gamma_{0}, \Gamma_{1}\right)^{\top}$ is surjective. Namely, let $\left(h_{0}, h_{1}\right)^{\top} \in \mathcal{H} \oplus \mathcal{H}$, where $h_{0}=\left\{h_{0 j}\right\}_{j=1}^{m}, h_{1}=\left\{h_{1 j}\right\}_{j=1}^{m}$ are vectors from $\mathbb{C}^{m}$. If $f \in \operatorname{dom}\left(H^{*}\right)$, then, by (4.2), $f=f_{H}+\sum_{j=1}^{m}\left(\xi_{0 j} e^{-r_{j}} \ln \left(r_{j}\right)+\xi_{1 j} e^{-r_{j}}\right)$. Let us put

$$
E_{0}:=\left(e^{-\left|x_{k}-x_{j}\right|} \ln \left(\left|x_{k}-x_{j}\right|+\delta_{k j}\right)\right)_{j, k=1}^{m}, \quad E_{1}:=\left(e^{-\left|x_{k}-x_{j}\right|}\right)_{k, j=1}^{m} .
$$

As above, invertibility of the matrix $E_{1}$ might be derived from the fact that the function $e^{-|x|}$ is strictly positive definite on $\mathbb{R}^{2}$ (see Example 3.13). Therefore, setting $\xi_{0}=\frac{1}{2 \pi} h_{0}$ and $\xi_{1}=E_{1}^{-1}\left(h_{1}-\frac{1}{2 \pi} E_{0} h_{0}\right)$, we get $\Gamma_{0} f=h_{0}$ and $\Gamma_{1} f=h_{1}$. Thereby, $(i i i)$ is proved. 
Analogously to the previous case, the following parametrization of the self-adjoint extensions is valid.

Proposition 5.2 Let $H$ be the minimal Schrödinger operator defined by $[1.2], \Pi=\left\{\mathcal{H}, \Gamma_{0}, \Gamma_{1}\right\}$ be a boundary triplet for $H^{*}$ defined by (5.5)-(5.6) and let the matrices $E_{0}, E_{1}$ be defined by (5.11). Then the set of self-adjoint extensions $\widetilde{H} \in$ Ext $_{H}$ is parameterized as

$$
\widetilde{H}=H_{\Theta}=H^{*}\left\lceil\left\{f=\sum_{j=1}^{m}\left(\xi_{0 j} e^{-r_{j}} \ln \left(r_{j}\right)+\xi_{1 j} e^{-r_{j}}\right)+f_{H} \in \operatorname{dom}\left(H^{*}\right):\left\{\Gamma_{0} f, \Gamma_{1} f\right\} \in \Theta\right\},\right.
$$

where $\Theta$ runs through the set of all self-adjoint linear relations in $\mathcal{H}$. Namely, $\Theta_{\infty}$ and $\Theta_{\mathrm{op}}$ are defined by

$$
\begin{aligned}
& \Theta_{\infty}=\left\{0, \mathcal{H}_{\infty}\right\}=\left\{\left\{0, E_{1} \xi_{1}^{\prime \prime}\right\}: \xi_{1}^{\prime \prime} \perp E_{1} \xi_{0}, \xi_{0} \in \mathcal{H}_{\mathrm{op}}\right\}, \\
& \Theta_{\mathrm{op}}=\left\{\left\{2 \pi \xi_{0}, E_{0} \xi_{0}+E_{1} \xi_{1}^{\prime}\right\}: \xi_{0} \in \mathcal{H}_{\mathrm{op}}, \xi_{1}^{\prime}=E_{1}^{-1}\left(2 \pi B \xi_{0}-E_{0} \xi_{0}\right)\right\},
\end{aligned}
$$

where $B=B^{*} \in\left[\mathcal{H}_{\mathrm{op}}\right]$. In particular, $\widetilde{H}=H_{\Theta}$ is disjoint with $H_{0}$ if and only if $\operatorname{dom}(\Theta)=\mathbb{C}^{m}$. In this case $\Theta=\Theta_{\mathrm{op}}=g r B$.

Remark 5.3 The $m$-parametric family $H_{X, \alpha}^{(2)}$ investigated in [3, Theorem 1.1.1] is parameterized by the diagonal matrices $\Theta=\Theta_{\mathrm{op}}=B_{\alpha}=\operatorname{diag}\left(\alpha_{1}, . ., \alpha_{m}\right), \quad \alpha_{j} \in \mathbb{R}$.

$$
H_{X, \alpha}^{(2)}=H^{*}\left\lceil\left\{f=\sum_{j=1}^{m} \xi_{0 j} e^{-r_{j}} \ln \left(r_{j}\right)+\sum_{k, j=1}^{m} b_{j k}(\alpha) \xi_{0 k} e^{-r_{j}}+f_{H}\right\},\right.
$$

where $\widetilde{B}=\left(b_{j k}(\alpha)\right)_{j, k=1}^{m}=E_{1}^{-1}\left(2 \pi B_{\alpha}-E_{0}\right)$.

Proposition 5.4 Let $H$ be the minimal Schrödinger operator and let $\Pi=\left\{\mathcal{H}, \Gamma_{0}, \Gamma_{1}\right\}$ be the boundary triplet for $H^{*}$ defined by (5.5)-(5.6). Then

(i) the Weyl function $M(\cdot)$ corresponding to the boundary triplet $\Pi$ is

$$
M(z)=\left(\frac{1}{2 \pi}\left(\psi(1)-\ln \left(\frac{\sqrt{z}}{2 i}\right)\right) \delta_{j k}+\widetilde{G}_{\sqrt{z}}\left(x_{j}-x_{k}\right)\right)_{j, k=1}^{m}, \quad z \in \mathbb{C}_{+},
$$

where $\psi(1)=\frac{\Gamma^{\prime}(1)}{\Gamma(1)}, \quad \widetilde{G}_{\sqrt{z}}(x)= \begin{cases}i / 4 H_{0}^{(1)}(\sqrt{z}|x|), & x \neq 0 ; \\ 0, & x=0 .\end{cases}$

(ii) the corresponding $\gamma(\cdot)$-field is given by

$$
\gamma(z)\left\{a_{j}\right\}_{j=1}^{m}=\sum_{j=1}^{m} a_{j} \frac{i}{4} H_{0}^{(1)}\left(\sqrt{z}\left|x-x_{j}\right|\right) .
$$

Proof. Combining (5.2) with (5.5)-(5.6) and taking into account expansion (5.1), we arrive at $(i)$ and $(i i)$.

Remark 5.5 Note that the Weyl function in the form (5.13) appears in [3, chapter II.4, Theorem 4.1]. In the case $m=1$, the Weyl function was also computed in [7, section 10.3].

\subsection{Spectrum of the self-adjoint extensions of the minimal Schrödinger operator}

As above, the following lemma holds.

Lemma 5.6 Let $H^{*}$ be defined by (5.3)-(5.4). Then $0 \notin \sigma_{p}\left(H^{*}\right)$.

Proof. The proof repeats the one in the 3D case. It only should be noted that the functions $\ln \left(r_{j}\right), j \in$ $\{1, . ., m\}$, are harmonic in $\mathbb{R}^{2} \backslash X$ and $f_{0} \in \operatorname{dom}\left(H^{*}\right)$ admits the representation

$$
f_{0}=\sum_{j=1}^{m} \xi_{0 j} \ln \left(r_{j}\right)+g, \quad \text { with } \quad g:=\sum_{j=1}^{m}\left(\xi_{0 j}\left(e^{-r_{j}}-1\right) \ln \left(r_{j}\right)+\xi_{1 j} e^{-r_{j}}\right)+f_{H} \in W_{\mathrm{loc}}^{2,2}\left(\mathbb{R}^{2}\right),
$$

where $\left(e^{-r_{j}}-1\right) \ln \left(r_{j}\right) \in W_{\text {loc }}^{2,2}\left(\mathbb{R}^{2}\right)$ for any $j \in\{1, . ., m\}$. 
Spectrum of the self-adjoint extensions of $H$ is described in the following theorem.

Theorem 5.7 Let $H$ be the minimal Schrödinger operator defined by (1.2), let $\Pi$ be the boundary triplet for $H^{*}$ defined by (5.5)-(5.6), and $M(\cdot)$ be the corresponding Weyl function defined by (5.13). Assume also that $H_{\Theta}=H_{\Theta}^{*} \in \operatorname{Ext}_{H}$ is defined by (5.12). Then the following assertions hold.

(i) The extension $H_{\Theta}$ has purely absolutely continuous non-negative spectrum of infinite multiplicity.

(ii) Point spectrum of the self-adjoint extension $H_{\Theta}$ consists of at most $m$ negative eigenvalues (counting multiplicities). Moreover, $z \in \sigma_{p}\left(H_{\Theta}\right) \cap \mathbb{R}_{-}$if and only if $0 \in \sigma_{p}(\Theta-M(z))$, i.e.,

$$
z \in \sigma_{p}\left(H_{\Theta}\right) \cap \mathbb{R}_{-} \Longleftrightarrow 0 \in \sigma_{p}\left(\Theta-\left(\frac{1}{2 \pi}\left(\psi(1)-\ln \left(\frac{\sqrt{z}}{2 i}\right)\right) \delta_{j k}+\widetilde{G}_{\sqrt{z}}\left(x_{j}-x_{k}\right)\right)_{j, k=1}^{m}\right) .
$$

The corresponding eigenfunctions $\psi_{z}$ have the form

$$
\psi_{z}=\sum_{j=1}^{m} a_{j} \frac{i}{4} H_{0}^{(1)}\left(\sqrt{z} r_{j}\right), \quad \text { where } \quad\left(a_{1}, . ., a_{m}\right)^{\top} \in \operatorname{ker}(\Theta-M(z)) .
$$

Pro of. The proof is similar to that of Theorem 4.7 It follows from (5.13) that

$$
M(x+i 0)=\left(\frac{1}{2 \pi}\left(\psi(1)-\ln \left(\frac{\sqrt{x}}{2 i}\right)\right) \delta_{j k}+\widetilde{G}_{\sqrt{x}}\left(x_{j}-x_{k}\right)\right)_{j, k=1}^{m}, \quad x \in \mathbb{R} .
$$

Note that, according to [27, chapter 7,§4],

$$
\operatorname{Im}\left(\widetilde{G}_{\sqrt{x}}\left(x_{j}-x_{k}\right)\right)=\frac{1}{4} J_{0}\left(\sqrt{x}\left|x_{j}-x_{k}\right|\right), \quad x \geq 0,
$$

where $J_{0}(\cdot)$ is the Bessel function. Combining this fact with (5.14), we get

$$
\operatorname{Im}(M(x+i 0))=\left(\frac{1}{4} J_{0}\left(\sqrt{x}\left|x_{j}-x_{k}\right|\right)\right)_{j, k=1}^{m}, \quad x \geq 0 .
$$

In a view of (3.3), $J_{0}(s x) \in \Phi_{2}, s>0$ (see Example 3.13), and therefore the matrix $\operatorname{Im}(M(x+i 0))$ is positive definite for $x \in \mathbb{R}_{+}$.

Remark 5.8 The description of absolutely continuous and point spectrum in the particular case of the family $H_{X, \alpha}^{(2)}$ was obtained in [3, Theorem 1.1.4].

\section{References}

[1] V. Adamyan, Nonnegative Perturbations of Nonnegative Self-adjoint Operators, Methods Funct.Anal.Topology 13 (2), 103-109 (2007).

[2] N.I. Akhiezer, The classical moment problem and some related questions of analysis (Fizmatlit, Moscow, 1961)(Russian). (English translation: Oliver and Boyd, Edunburgh, 1965).

[3] S. Albeverio, F. Gesztesy, R. Hoegh-Krohn, H. Holden, Solvable Models in Quantum Mechanics, Texts and Monographs in Physics (Springer, Berlin-New York, 1988).

[4] S. Albeverio, P. Kurasov, Singular Perturbations of Differential Operators (London Mathematical Society Lecture Note Series) 271 (Cambridge University Press, Cambridge, 1999).

[5] Yu. Arlinskii, E. Tsekanovskii, The von Neumann Problem for Nonnegative Symmetric Operators, Integr. Eq. Oper. Theory 51, 319-356 (2005).

[6] R. Askey, Radial characteristic functions, Tech. Report No. 1262 (Math. Research Center, University of WisconsinMadison, 1973).

[7] M.S. Ashbaugh, F. Gesztesy, M. Mitrea, G. Teschl, Spectral Theory for Perturbed Krein Laplasians in Nonsmooth Domains, Adv. Math. 51, 1372-1467 (2010).

[8] F.A.Berezin, L.D.Faddeev, Remark on the Schrödinger equation with singular potential, Dokl.Acad.Sci. USSR 137, 1011-1014 (1961)(Russian).

[9] J.F. Brasche, M. Malamud, H. Neidhardt, Weyl function and spectral properties of self-adjoint extensions, Integr. Eq. Oper. Theory 43, 264-289 (2002). 
[10] J. Behrndt, M. Malamud, H. Neidhardt, Scattering matrices and Weyl functions, Proc. London Math. Soc. 97, 568-598 (2008).

[11] S. Bochner, Monotone Funktionen, Stieltjessche Integrale und harmonische Functionen, Math. Ann. 108, 1933.

[12] V.A. Derkach, M.M. Malamud, Generalized resolvents and the boundary value problems for hermitian operators with gaps, J. Funct. Anal. 95, 1-95 (1991).

[13] V.A. Derkach, M.M. Malamud, The extension theory of Hermitian operators and the moment problem, J. Math. Sci. (New York), 73, 141-242 (1995).

[14] G. Fasshauer, Meshfree approximation methods with MATLAB (World Scientific Publishers, 2007).

[15] G. M. Fikhtengoltz, Course of differential and integral calculus, Vol. III (Gos. izd. fiz.-mat. lit., Moscow, 1959) (Russian).

[16] F. Gesztesy, K. A. Makarov, and M. Zinchenko, Essential closures and AC spectra for reflectionless CMV, Jacobi, and Schrödinger operators revisited, Acta Appl. Math. 103 (3), 315-339 (2008).

[17] D. Gilbarg, Neil S. Trudinger, Elliptic Partial Differential Equations of Second Order (Springer, New York, 1988).

[18] B.I. Golubov, On Abel-Poisson type and Riesz means, Analysis Math. 7, 161-184 (1981).

[19] V.I. Gorbachuk, M.L. Gorbachuk, Boundary Value Problems for Operator Differential Equations (Naukova Dumka, Kiev, 1984) (Russian). (English translation: Mathematics and its Applications (Soviet Series) 48 Kluwer Academic Publishers Group, Dordrecht, 1991).

[20] S. Hassi, S. Kuzhel, On symmetries in the theory of singular perturbations, J. Funct. Anal. 256, 777-809 (2009).

[21] A.N. Kochubei, Elliptic operators with boundary conditions on a subset of measure zero, Funct. Anal. Appl. 16 (2), 137-139 (1982).

[22] V.E. Lyantse, H.B. Majorga, On the Theory of One-Point Boundary-Value Problem for Laplas Operator, Function Theory, Funct. Anal. and Appl. 38, 84-91 (1982)(Russian).

[23] M. Malamud, H. Neidhardt, On the unitary equivalence of absolutely continuous parts of self-adjoint extensions, J. Funct. Anal. 260 (3), 613-638 (2011).

[24] V.P. Mikhailov, Partial differential equations, (Nauka, Moscow, 1976) (Russian).

[25] M. A. Naimark, Linear differential operators, (Nauka, Moscow, 1969) (Russian).

[26] O. Ogurisu, On the number of negative eigenvalues of a multi-dimensional Schrödinger operator with point interactions, Methods Funct. Anal. Topology 16 (4), 383-392 (2010).

[27] F. Olver, Introduction to Asymptotic Methods and Special Functions (Nauka, Moscow, 1978)(Russian).

[28] A. Posilicano, Self-Adjoint Extensions of Restrictions, Operators and Matrices 2 (4), 483-506 (2008).

[29] M. Reed, B. Simon, Methods of modern mathematical physics, vol. IV, Analysis of operators (Academic Press, New York, 1978).

[30] I. J. Schoenberg, Metric spaces and completely monotone functions, Ann. Math. 39, 811-841 (1938).

[31] I.J. Schoenberg, Metric spaces and positive definite functions, Trans. Amer. Math. Soc. 44, 522-536 (1938).

[32] R. M. Trigub, A criterion for a characteristic function and Polyá type criterion for radial functions of several variables, Theory Probab. Appl. 34, 738-742 (1989).

[33] H. Wendland, Scattered Data Approximation (Cambridge University Press, Cambridge, 2005).

[34] V.P. Zastavnyi, Positive definite functions depending on a norm, Dokl. Russian Acad. Nauk, 325, 901-903 (1992)(Russian). (English translation: Russian Acad. Scie. Dokl. Math. 46 (1), 112-114 (1993)).

[35] V.P. Zastavnyi, On positive definiteness of some functions, J. Multiv. Anal. 73, 55-81 (2000). 